\title{
THE UNIQUE CYTOARCHITECTURE AND WIRING OF THE HUMAN DEFAULT MODE NETWORK
}

\author{
Casey Paquola ${ }^{1,2}$, Margaret Garber ${ }^{1}$, Stefan Frässle ${ }^{3}$, Jessica Royer ${ }^{1}$, Shahin Tavakol ${ }^{1}$, Raul Rodriguez- \\ Cruces $^{1}$, Elizabeth Jeffries ${ }^{4}$, Jonathan Smallwood ${ }^{5}$, Boris Bernhardt ${ }^{1}$ \\ ${ }^{l}$ Montreal Neurological Institute \& Hospital, McGill University, Montréal, Quebec, Canada \\ ${ }^{2}$ INM-1, Forschungszentrum Jülich, Jülich, Germany \\ ${ }^{3}$ Translational Neuromodeling Unit (TNU), University of Zurich \& ETH Zurich, Zurich, Switzerland \\ ${ }^{4}$ Department of Psychology, University of York, York, York, United Kingdom \\ ${ }^{5}$ Department of Psychology, Queen's University, Kingston, Ontario, Canada
}

\begin{abstract}
It is challenging to specify the role of the default mode network (DMN) in human behaviour. Contemporary theories, based on functional magnetic resonance imaging (MRI), suggest that the DMN is insulated from the external world, which allows it to support perceptually-decoupled states and to integrate external and internal information in the construction of abstract meanings. To date, the neuronal architecture of the DMN has received relatively little attention. Understanding the cytoarchitectural composition and connectional layout of the DMN will provide novel insights into its role in brain function. We mapped cytoarchitectural variation within the DMN using a cortical type atlas and a histological model of the entire human brain. Next, we used MRI acquired in healthy young adults to explicate structural wiring and effective connectivity. We discovered profound diversity of DMN cytoarchitecture. Connectivity is organised along the most dominant cytoarchitectural axis. One side of the axis is the prominent receiver, whereas the other side remains more insulated, especially from sensory areas. The structural heterogeneity of the DMN engenders a network-level balance in communication with external and internal sources, which is distinctive, relative to other functional communities. The neuronal architecture of the DMN suggests it is a protuberance from the core cortical processing hierarchy and holds a unique role in information integration.
\end{abstract}




\section{INTRODUCTION}

Complex behaviour benefits from parallel distributed processing in brain networks (Felleman and Van Essen, 1991; Goldman-Rakic and Schwartz, 1982; Mesulam, 1990; Rumelhart and McClelland, 1987, 1986). This architecture allows for the simultaneous and varied consideration of multiple sources of information (Cisek, 2012; Felleman and Van Essen, 1991; Mesulam, 1990). The roles of certain networks are well-defined, while others remain elusive. Arguably, none are so elusive as the default mode network (DMN) (Buckner et al., 2008; Raichle, 2015). Initial observations were that DMN regions decrease activity during externally oriented tasks, thus behaving in a somewhat diametral way to sensory and attentional systems (Fox et al., 2005; Raichle et al., 2001; Shulman et al., 1997). Subsequently, several lines of research have demonstrated an active role of the DMN in self-generated cognitive operations that draw on memory (Li et al., 2021; McCormick et al., 2014; Sestieri et al., 2011). Examples include self-referential cognition (Davey et al., 2016; Molnar-Szakacs and Uddin, 2013; Spreng et al., 2010), complex decision making (Murphy et al., 2019, 2018), social cognition (Alcalá-López et al., 2018; Frith and Frith, 2012), and narrative comprehension (Mar, 2011; Simony et al., 2016). As these complex behaviours share a dependence on integration of information from internal as well as external sources (Brandman et al., 2021), contemporary theories have also postulated that balancing internal and external information may be a core aspect of DMN function (Buckner et al., 2008; Smallwood et al., 2021a; Yeshurun et al., 2021).

Complementing behavioural accounts, the functional role of networks such as the DMN may be understood with respect to their neuronal architecture. The DMN is composed of several areas distributed across the frontal, parietal and temporal cortex. Histological studies show that these regions vary in their cellular make-up (Brodmann, 1903; Von Economo and Koskinas, 1925). Even within a circumscribed region of the DMN, such as the inferior parietal lobe or medial prefrontal cortex, distinct cytoarchitectural areas can exist (Bludau et al., 2014; Caspers et al., 2008, 2006). Thus, the DMN is cytoarchitecturally heterogenous, although the manner and influence of this cytoarchitectural variation is not known. One appealing candidate to link cytoarchitecture to function is laminar differentiation (Barbas, 2015, 1986; Barbas and RempelClower, 1997). The clarity of cortical layers as well as the prominence of a granular layer IV, also referred to as "granularity", decreases with synaptic distance from primary sensory areas (Mesulam, 1998; Pandya and Yeterian, 1985). These cytoarchitectural changes present as a gradient running across the cortical surface (García-Cabezas et al., 2020; Paquola et al., 2019b), which is commonly termed the "sensory-fugal" axis, as it mirrors a shift in receptiveness from the external world to the internal milieu Thus, cortical types, which discretise the sensory-fugal gradient of laminar elaboration (García-Cabezas et al., 2020; Von Economo and Koskinas, 1925), can index coupling of neural responses to the external world. Further characterisation of DMN cytoarchitecture is possible with an ultra-high-resolution histological reconstruction of the human brain (Amunts et al., 2013). BigBrain, the first dataset of this kind (Amunts et al., 2013), allows computational analysis of cytoarchitecture across the entire human brain. It may be that the dominant pattern of cytoarchitectural differentiation in the DMN is the gradient of laminar elaboration, or an altogether distinct pattern.

In the context of the whole cortex, the DMN occupies a distinct topographic position (Smallwood et al., 2021a). Parts of the DMN are situated most distally from primary sensory and motor areas (Leech et al., 2014; Margulies et al., 2016). The separation from primary sensory areas reinforces that the DMN is involved in functions that require integration between distinct sources, while also being relatively insulated from the influence of one specific input. Multi-variate decompositions of resting state fMRI support this notion, illustrating antipodal functional dynamics of the DMN and primary sensory areas (Hong et al., 2019; Margulies et al., 2016; Sepulcre et al., 2012). Based on these results, it was hypothesised that the DMN is the apex of the sensory-fugal hierarchy. The validity of this hypothesis may be evaluated based on physical 
embedding of the DMN within the whole-brain connectome. However, studies mapping white matter tracts of the DMN have thus far focused on specific fiber bundles that interconnect its different regions (Alves et al., 2019; Figley et al., 2015; Greicius et al., 2009; Horn et al., 2014; Khalsa et al., 2014; Sandhu et al., 2020; Van Den Heuvel et al., 2008; Van Oort et al., 2014). Thus, how subunits of the DMN contribute to both integration and perceptually-decoupled cognition remains unclear. Extant functional connectivity studies show regionally variable coherence of the DMN with other large-scale networks, especially attention networks (Anderson et al., 2011; Braga et al., 2013; Dixon et al., 2017; He et al., 2009; Uddin et al., 2009), which suggest complementary functions of subunits towards network-level roles. Crucially, dynamic models of cortical function offer enhanced sensitivity to regional differences (Dixon et al., 2017) and will allow inference on the directionality of information flow to and from the DMN.

Revealing the cytoarchitectural composition and connectional layout of the DMN will provide novel insights into the structural basis of DMN function. To this end, we mapped cytoarchitectural variation within the DMN using a cortical type atlas (García-Cabezas et al., 2020; Von Economo and Koskinas, 1925) and a histological model of the entire human brain (Amunts et al., 2013) (Figure 1). We aimed to determine the spatial pattern and specific histological features of cytoarchitectural differentiation within the DMN. Next, we used magnetic resonance imaging (MRI) acquired in healthy young adults (Royer et al., 2021; Van Essen et al., 2013) to explicate structural wiring and effective connectivity. In both modalities, we examined the balance of communication from the DMN to different cortical types, where granularity of the cortical type proxies coupling to external sources. Then, we tested whether cytoarchitectural variations within the DMN account for the balance in connectivity. Finally, we evaluated whether cytoarchitectural heterogeneity and connectional balance were unique properties of the DMN, by comparing its features to other large-scale functional brain networks.

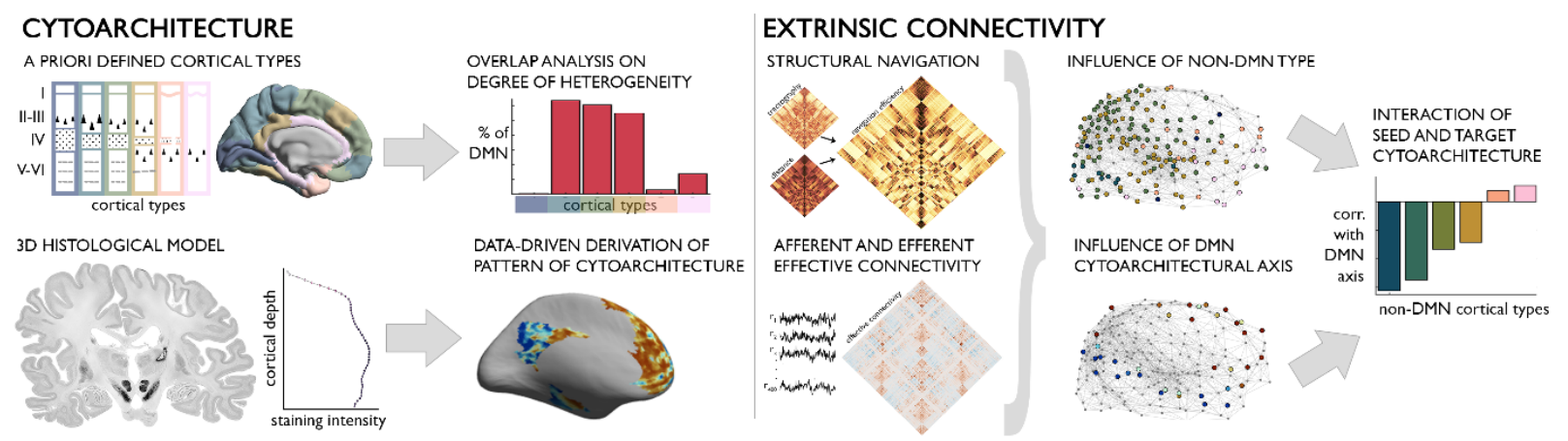

Figure 1: Overview of core analyses. Left Characterising cytoarchitecture of the DMN using a priori defined cortical types (García-Cabezas et al., 2020; Von Economo and Koskinas, 1925) and a histological model of the entire human brain (Amunts et al., 2013). Complementary atlas overlap and unsupervised machine learning procedures were used to evaluate the degree and pattern of cytoarchitectural heterogeneity, respectively. Right The position of the DMN in the whole brain network was assessed structurally and functionally using navigation efficiency (Seguin et al., 2019) and directed effective connectivity (Frässle et al., 2021b). Then, the relation of cytoarchitectural heterogeneity to connectivity were principally determined with three tests, which used properties of seed/target regions to explain variation in the strength of connectivity. The properties were cortical type for nonDMN regions and data-driven cytoarchitectural axis values for DMN regions. The main effect of each were tested, as well as their interaction.

\section{RESULTS}

The DMN shows marked cytoarchitectural heterogeneity

Using established maps of cytoarchitectural differentiation (García-Cabezas et al., 2020; Paquola et al., 2019b; Von Economo and Koskinas, 1925), we found that the DMN harbours all six cortical types, signifying marked cytoarchitectonic heterogeneity (Figure 2B). Chi-squared tests showed that cortical 
bioRxiv preprint doi: https://doi.org/10.1101/2021.11.22.469533; this version posted November 22, 2021. The copyright holder for this preprint (which was not certified by peer review) is the author/funder, who has granted bioRxiv a license to display the preprint in perpetuity. It is made available under aCC-BY-NC-ND 4.0 International license.

types were not equally represented within the DMN, however (types: $\chi^{2}=1497, \mathrm{p}<0.001$ ). By comparing the proportions of cortical types in the DMN to 1000 randomly rotated versions of the atlas, which preserve the size and shape of the DMN (Alexander-Bloch et al., 2018), we found that the DMN significantly overrepresents eulaminate-I $\left(18 \%\right.$ increase; $\left.\mathrm{p}_{\text {spin }}=0.006\right)$ and under-represents the koniocortical type $(7 \%$ decrease, $\left.p_{\text {spin }}=0.011\right)$ (Supplementary Figure 1).

A | Canonical default mode network (DMN)
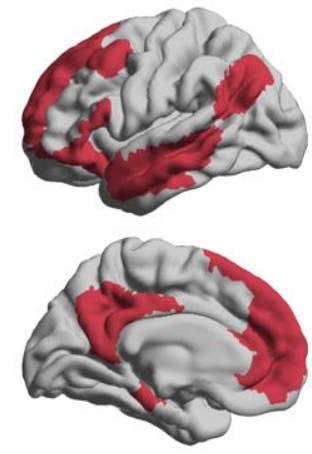
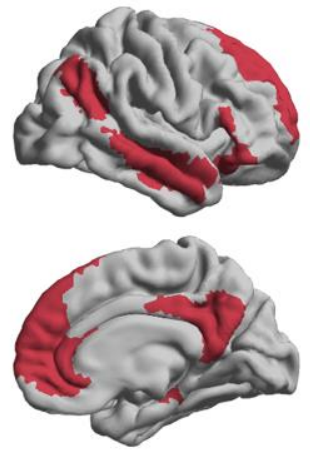

B | Distribution of cortical types
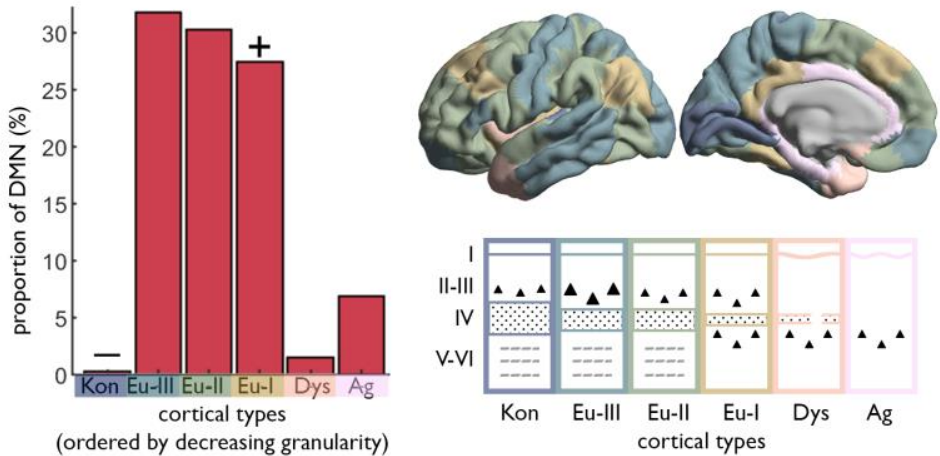

C | Cytoarchitectural gradient within the DMN
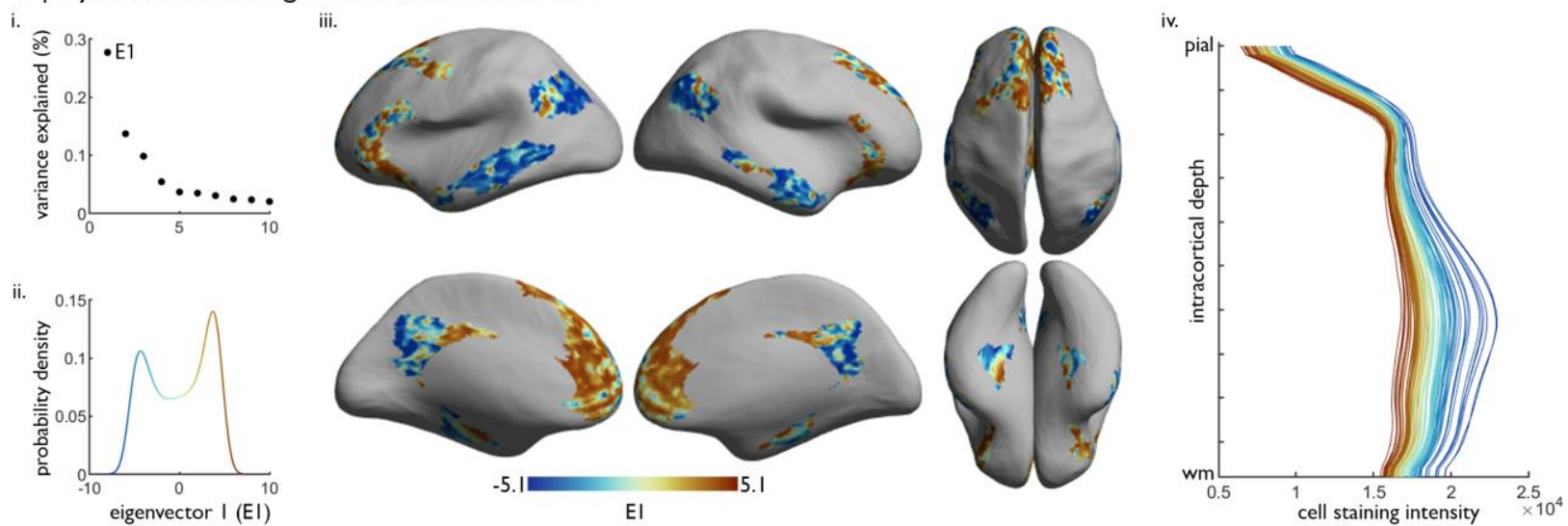

D | Distinct patterns of cytoa
i. Distribution of $\mathrm{E} 1$ within subregions
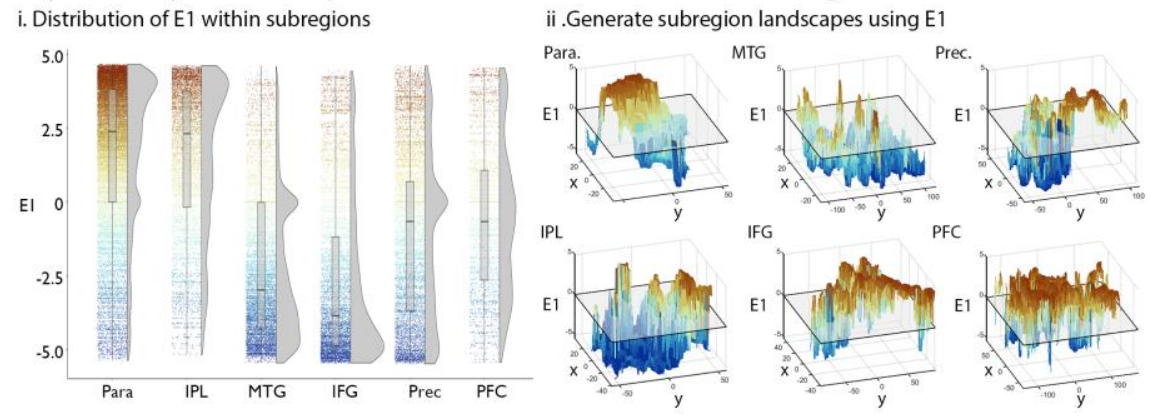

iii. Number of intersections of $E 1=0$ plane $(n 0)$

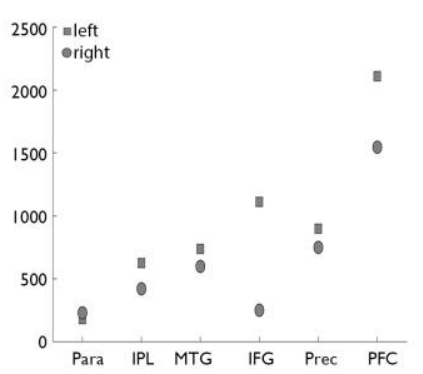

Figure 2: Cytoarchitectural heterogeneity of the DMN. A) DMN defined by (Yeo et al., 2011). B) Histogram depicts the frequency of cortical types within the DMN, with + indicative of significant over-representation and - for under-representation, relative to a spatially rotated null model. Cortical types are shown on the surface. The schematic highlights prominent features that vary across cortical types, including the location/size of largest pyramidal neurons (triangles), thickness of layer IV, existence of sublayers in V-VI (grey dashed lines), regularity of layer I/II boundary (straightness of line). Kon=koniocortical. Eul=eulaminate. Dys=dysgranular. Ag=agranular. C) i. Scatterplot shows approximate variance explained of affinity matrix by each eigenvector, which reflects variation in cytoarchitecture. ii. Probability density plot shows a bimodal distribution of E1 values. iii. E1 projected onto the inflated BigBrain surface. iv. Staining intensity profiles averaged within 100 bins of E1 and coloured by E1 position show the shift from skewed to flat profiles along the cytoarchitectural axis. D) i. Raincloud plot shows the distribution of E1 within 
circumscribed subregions of the DMN. Para: parahippocampus. IPL: inferior parietal lobule. MTG: middle temporal gyrus. IFG: inferior frontal gyrus. Prec: precuneus. PFC: prefrontal cortex. ii. The topography of E1 on the cortex shown as a 3D surface plot, with $\mathrm{E} 1$ as the $\mathrm{z}$-axis. For visualisation purposes, the $\mathrm{x}$ - and $\mathrm{y}$-axes are defined by isomap flattening of the subregion. In the analyses, the spatial information is determined by edge relationships on the surface mesh. iii. Scatterplot shows the number of intersections of the $\mathrm{E} 1=0$ plane for each subregion. Few intersections indicate the slope of the landscape is more unidirectional, while many intersections suggest a bumpier landscape.

We mapped cytoarchitectural variability within the DMN by measuring intracortical variations in cell body staining from BigBrain, a high-resolution 3D reconstruction of a post mortem human brain (Amunts et al., 2015). A total of 71,576 staining intensity profiles, oriented in the direction of cortical columns, were used to quantify depth-wise variations in cell density and soma size. The principle axis of variation in cytoarchitecture was revealed by diffusion map embedding, a nonlinear manifold learning technique (Coifman et al., 2005; Margulies et al., 2016; Vos de Wael et al., 2020). The first eigenvector (E1) explained $\sim 27 \%$ of variance and illustrated a marbled pattern of cytoarchitectural differentiation within the DMN (Figure 2C). The E1 pattern is distinct to the gradient of laminar elaboration that is captured by the cortical types. Both are anchored by koniocortex on one side and agranular cortex on the other, but they differ in the ordering of eulaminate and dysgranular cortex (Supplementary Figure 1).

Each subregion of the DMN comprises a broad range of E1, which indicates intra-regional cytoarchitectural diversity (Figure 2Di). The spatial pattern of E1 differs between subregions, however. On the one hand, in the parahippocampal region, E1 values vary relatively smoothly across the surface, exhibiting a cytoarchitectural gradient (i.e. a graded, unidirectional change in E1 values). On the other hand, in the lateral prefrontal cortex, E1 transitions between extremes periodically along the surface, representing interdigitation of different cytoarchitectural forms. Such interdigitation is also observed in inferior frontal gyrus, inferior parietal lobule and the medial temporal gyrus. Between these extreme cases, cingulate-linked regions (i.e. medial prefrontal cortex and precuneus) exhibit gradients with striations. E1 values generally decrease with distance from the cingulate, but there are intermittent deviations from the gradient trend. The striations are not clearly aligned with curvature (spatial correlation, $r=-0.08$ ). We quantified differences in the spatial patterns by calculating roughness parameters (Gadelmawla et al., 2002a) of the cortical surface, using E1 as the texture or elevation of the cortical landscape. In terms of spatial roughness parameters, the number of intersections of the $\mathrm{E} 1=0$ plane (n0) varied most substantially across subregions $(74 \%$ variation, see Supplementary Table 1 for all parameters). Additionally, greater variation was observed across spatial parameters (median variation=41\%) than amplitude parameters (median variation=15\%), which complements our qualitative assessment that subregions differ in terms of the spatial patterning of cytoarchitectural differentiation more than the total cytoarchitectural content.

E1 captures changes in the intracortical staining intensity profiles, which capture depth-wise variations in the density and size of cell bodies. Areas with lower E1 values exhibit higher overall staining intensity, with a noticeable peak at mid-depths, whereas areas with higher E1 values show overall lower staining intensity with a flatter profile and more limited differentiation across depths (Figure 2C right). To evaluate these changes quantitatively, we characterised the shape of each staining intensity profile based on intensity at each depth as well as the central moments (mean, SD, skewness and kurtosis) (Paquola et al., 2020; Schleicher et al., 1999; Zilles et al., 2002). Then, we used random forest regression, an ensemble learning algorithm that is well-suited to feature selection (Strobl et al., 2008), to identify staining intensity profile features (depth-specific intensity or central moments) that explain E1. A reduced model with profile skewness and staining intensity at approximately $10 \%$ and $12 \%$ depth provided strong explanatory power of $\mathrm{E} 1$ (mean squared error $=0.04, \mathrm{R}^{2}=0.80$ ) (Supplementary Figure 2). Skewness, the most salient feature that changes along E1, relates to the relative staining in supra $v s$ infragranular layers. Lower E1 values correspond to a high skewness, whereas higher E1 values reflect a flatter profile (skewed-flat axis). This 
emphasises the importance of distribution of cells across multiple layers in defining cytoarchitecture (Paquola et al., 2020). In addition, intensity at 10/12\% depth, aligns with the layer I/II border, depicting a more specific aspect of cytoarchitectural differentiation in the DMN.

\section{Divergence of structural connectivity along the cytoarchitectural axis}

Next, we expanded our neuroanatomical study of the DMN from the intrinsic neuronal architecture to its extrinsic connectivity. We constructed a group-consensus structural connectome of 400 functionallydefined parcels (Schaefer et al., 2018) using diffusion weighted MRI tractography from 40 healthy adults (Figure 3Ai). Within each hemisphere, we calculated parcel-to-parcel navigation efficiency based on the structural connectome (Figure 3Aii). Navigation efficiency relates to distance travelled between a seed and a target along the structural connectome and previous work has shown navigation closely approximates invasive tract tracing connectomes in non-human primates (Seguin et al., 2019). Higher navigation efficiency indexes stronger communication with lower wiring cost.

\section{A | Navigation efficiency of the DMN within the structural connectome}
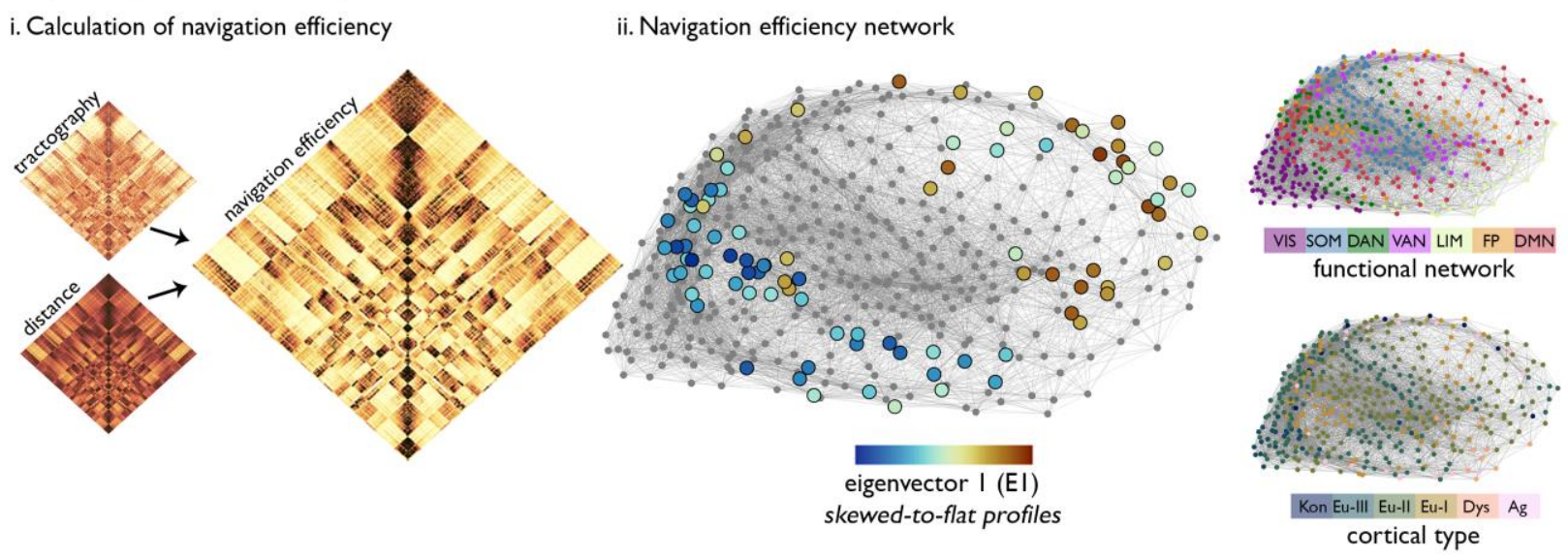

B | Navigation efficiency of the DMN to different cortical types

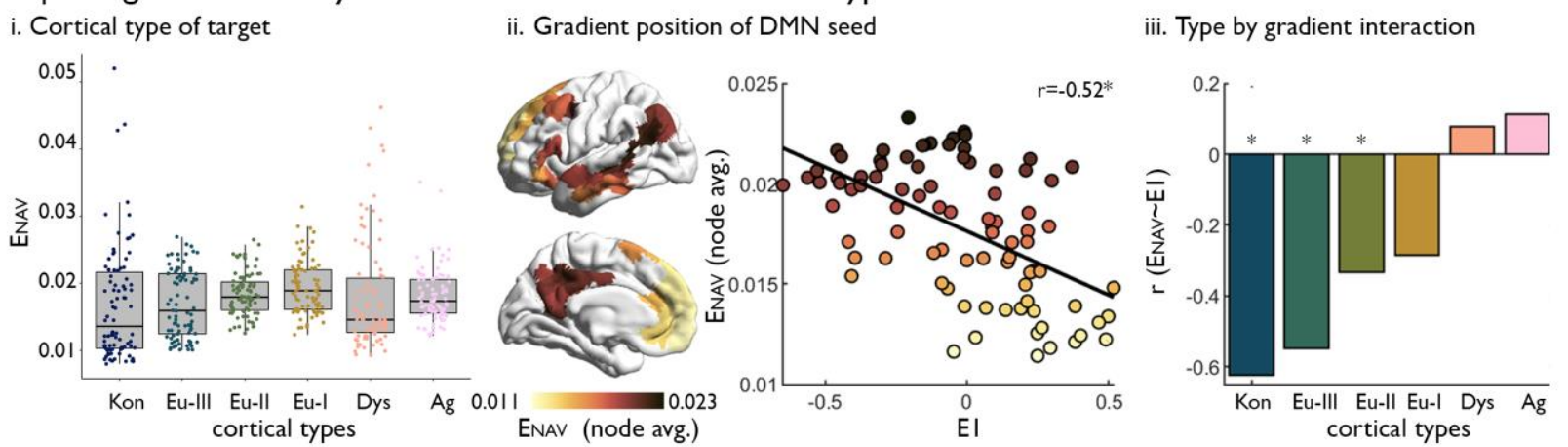

Figure 3: DMN structural connectivity organised with respect to cytoarchitecture. A) i. Navigation efficiency (Enav) was calculated from step-wise progression along the tractography-based connectome, where each step is determined by spatial proximity to the target node. . ii. Graph representation of the Enav connectome within the left hemisphere. The matched representations depict E1 (within DMN), functional networks, and cortical types . B) i. Type-effect: Boxplots show depict a striking balance in the average Enav of each DMN parcel across cortical types. ii. Axis-effect: Cortical surfaces show the average Enav of each DMN parcel. Scatterplot shows that DMN nodes lower on E1 have higher average Enav.iii. Interaction-effect: Bar plots shows the linear correlation coefficient ( $\mathrm{r}$ ) of E1 value with type-average Enav. The significant (*) negative $\mathrm{r}$ values indicate that DMN nodes lower on E1 have higher Enav to granular cortical types [koniocortex (Kon) and eulaminate (Eu) III-II] 
We focused on extrinsic connectivity of the DMN to discern its position in the whole brain connectome. We examined the relationship of cytoarchitecture to regional differences in DMN circuitry by labelling DMN seeds by E1 and labelling non-DMN targets by cortical type. Based on a multiple linear regression model, the cytoarchitectural features of seeds and targets together account for approximately $19 \%$ of variance in extrinsic navigation efficiency of the DMN (adjusted $\mathrm{R}^{2}=0.188, \mathrm{p}<0.001$ ). In post hoc analyses, we found navigation efficiency from the DMN to each type was relatively similar, as average navigation efficiency to each type did not significantly differ from null spin models $\left(0.44<\mathrm{p}_{\text {spin }}<0.53\right.$, Figure 3Bi). In contrast, we observed a significant decrease in average navigation efficiency along E1 ( $\mathrm{r}=-0.52, \mathrm{p}_{\text {spin }}=0.001$, Figure 3Bii). Additionally, we observed an interaction of E1 value of DMN seeds with the cortical type of non-DMN targets $\left(\mathrm{t}_{512,516}=7.94, \mathrm{p}<0.001\right)$. Navigation efficiency to the most granular types decreased along E1 (koniocortical/eulaminate III/eulaminate II; $\mathrm{r}=-0.63 /-0.55 /-0.33, \mathrm{p}_{\text {spin }}<0.025$ ), while navigation efficiency to less granular types was not significantly associated with E1 (eulaminate I/dysgranular/agranular; $r=-0.28 / 0.08 / 0.11, \mathrm{p}_{\text {spin }}>0.025$, Figure 3Biii). We did not observe this pattern for connectivity within the DMN (Supplementary Figure 3). Furthermore, the detected pattern of extrinsic connectivity was specific to the E1 and was not observed when defining DMN cytoarchitecture by cortical types or lower eigenvectors (Supplementary Figure 3). Together, this shows that structural connectivity of the DMN to other brain regions is organised along E1, particularly for more granular types that have higher navigation efficiency to DMN areas with lower E1 positions. The skewed aspect of E1 is thus structurally poised to integrate signals from a large cortical territory.

\section{Cytoarchitecturally-linked functional dynamics of the DMN}

The structural analyses demonstrate the potential for equipoised communication of the DMN with all cortical types as well as the organisation of extrinsic connectivity of the DMN according to cytoarchitectural differentiation. To assess directed information flow to and from the DMN, we modelled the effective connectivity of the whole cortex (400 nodes) using resting state fMRI and regression dynamic causal modelling [rDCM, (Frässle et al., 2017), Figure 4A]. rDCM is a scalable generative model of effective connectivity, and recent work demonstrated the ability of this approach to detect regional differences in afferent and efferent connectivity within and between large-scale functional networks (Frässle et al., 2021b).

We divided the effective connectivity matrix into an afferent component, reflecting input to the DMN from non-DMN areas, and an efferent component, reflecting output of the DMN to non-DMN areas. The impact of cytoarchitecture on connectivity strength was measured in two ways, related to the cortical type of nonDMN areas and E1 position of DMN areas. Based on a multiple linear regression model, these cytoarchitectural features together account for approximately $25 \%$ of variance in afferent connectivity (adj. $\left.\mathrm{R}^{2}=0.246, \mathrm{p}<0.001\right)$ and $5 \%$ of variance in efferent connectivity (adj. $\left.\mathrm{R}^{2}=0.058, \mathrm{p}<0.001\right)$, suggesting that they mainly determine inflow into the DMN. We compared type-average strength to 10,000 null models with permuted cortical type assignments, thus gauging whether type-average strength is significantly higher or lower than may be expected from the size and distribution of that cortical type. Afferent connectivity was enhanced from eulaiminate-I $\left(\mathrm{p}_{\text {spin }}<0.001\right)$, while efferent connectivity exhibited no clear preference for type (Figure 4B left). Next, we examined whether connectivity strength was related to the E1 values across the DMN. Input to the DMN was stronger towards the skewed aspect of E1 $\left(r=-0.41, p_{\text {spin }}<0.010\right)$, whereas output of the DMN was more balanced across E1 ( $\left.\mathrm{r}=0.05, \mathrm{p}_{\text {spin }}<0.362\right)$ (Figure 4B centre). Finally, we tested for an interaction between cytoarchitecture of seed and target by calculating the correlation of connectivity with the E1 separately for each cortical type. Afferent connectivity was consistently stronger at the lower anchor of E1, regardless of the cortical type of the seed $\left(-0.45<\mathrm{r}<-0.22, \mathrm{p}_{\mathrm{spin}}<0.004\right)$, whereas no association to E1 was observed for type-specific efferent connectivity $(-0.06<\mathrm{r}<-0.16$, 
$\left.0.129<\mathrm{p}_{\text {spin }}<0.410\right)$ (Figure 4B right). The analyses show input to the DMN is focused upon the skewed aspect of E1, corresponding to lower E1 values, and output of the DMN is broadcast to a wide cytoarchitectural spectrum.

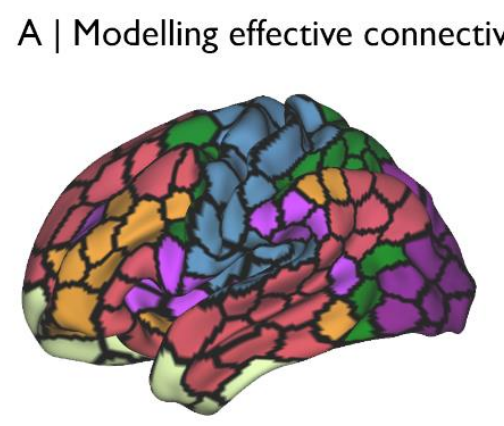

400 region parcellation

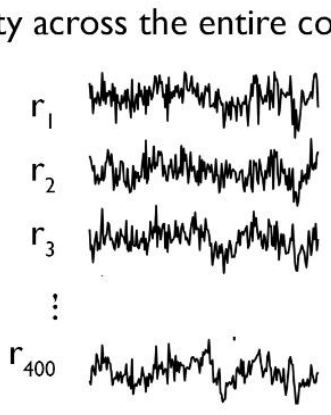

rsfmri BOLD timseries
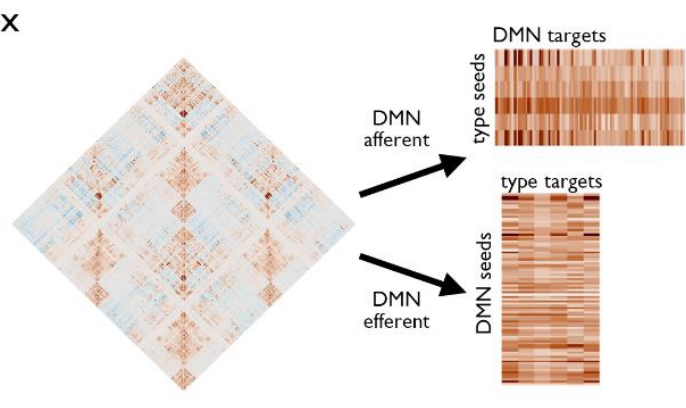

effective connectivity $(E C)$

B | Relation of cortical type and cytoarchitectural gradient to effective connectivity
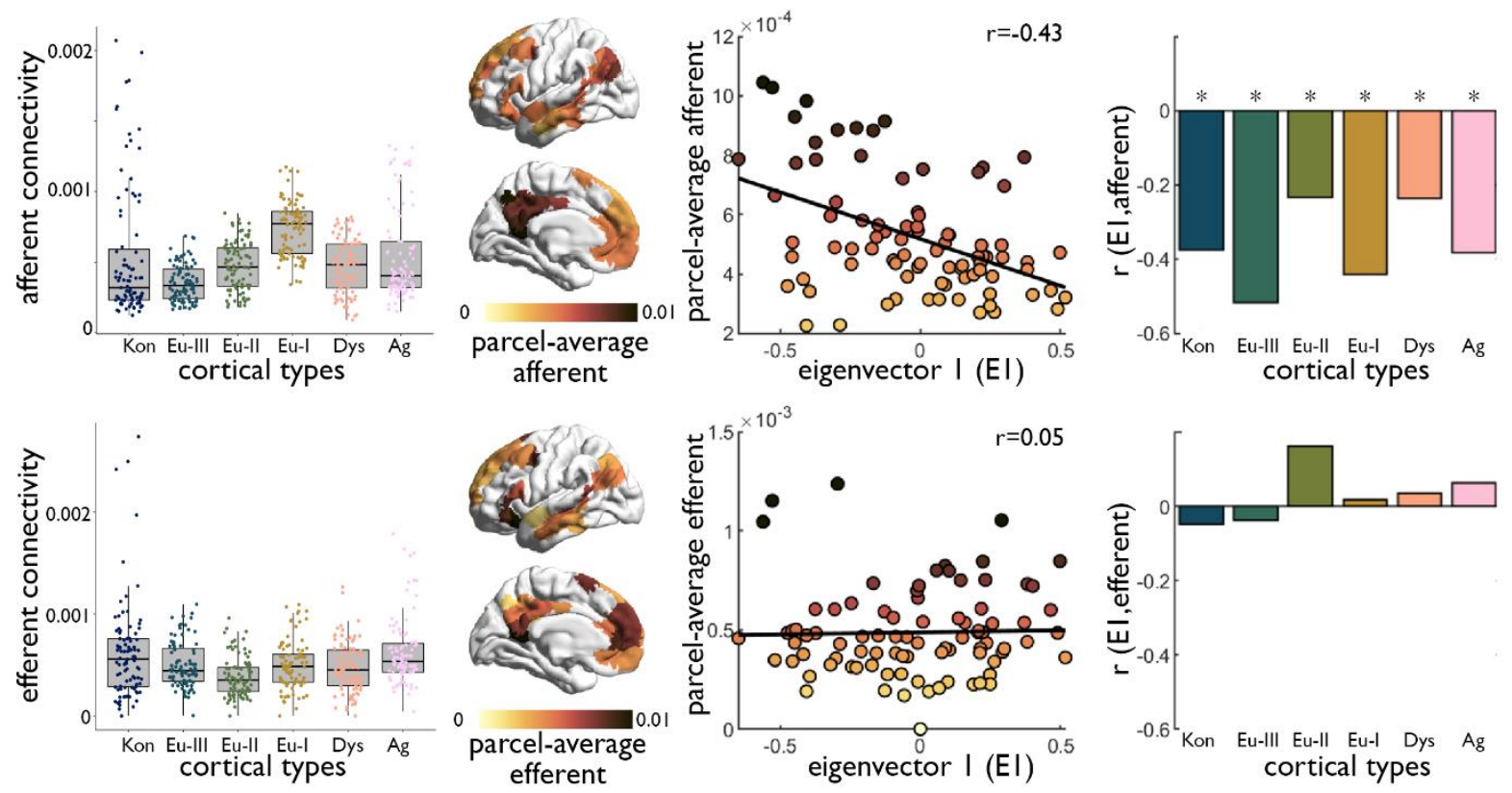

Figure 4: Relation of cytoarchitecture to effective connectivity $(E C)$ of the default mode network $(D M N)$. A) Methodological overview. Resting state fMRI (rsfmri) timeseries were extracted from 400 cortical regions (Schaefer et al., 2018) and EC was modelled using regression dynamic causal modelling. The EC matrix was split into afferent and efferent connectivity matrices, relative to the DMN. Effective connectivity of non-DMN parcels were averaged by cortical type. B) Left Boxplots show typeaverage effective connectivity with the DMN, where each point reflecting a DMN parcel. Centre Surface plots show the average effective connectivity of each DMN parcel, taken across all non-DMN parcels. This represents the y-axis in the correlation with parcel-wise values of the DMN cytoarchitectural axis in the scatterplot. Right Bar plots show the correlation coefficient typeaverage effective connectivity with the cytoarchitectural axis. Asterisks indicate $\mathrm{p}_{\mathrm{spin}}<0.004$, (ie: Bonferroni correction of $\alpha=0.05$ for six two-sided tests).

\section{Unique cellular and connectional architecture of the DMN}

Finally, we asked whether cytoarchitectural heterogeneity and balancing communication across cortical types were unique to the DMN or whether these features are present in other brain networks. While each functional network harbours multiple cortical types (Figure 5Ai), the distribution of types differs 
significantly between the DMN and other functional networks, indicating a unique cytoarchitectural makeup of the DMN $\left(3805>\chi^{2}>34235, \mathrm{p}<0.001\right.$, Supplementary Table 2). We also measured cytoarchitectural heterogeneity based on pair-wise correlation of staining intensity profiles within each network, relative to a null model that represents a homogenous network. Kullback-Leibler (KL) divergence from the null model indicated highest cytoarchitectural heterogeneity in the DMN, closely followed by the ventral attention network (Figure 5Aii).
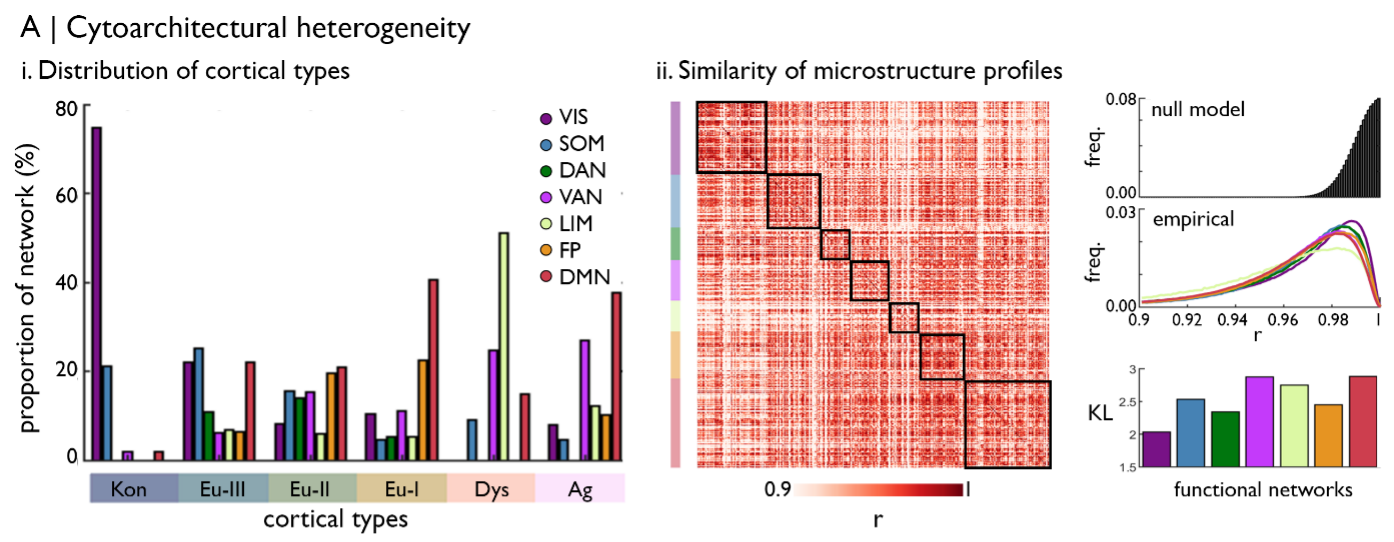

B | Balance of navigation efficiency to cortical types i. ENAv to each cortical type
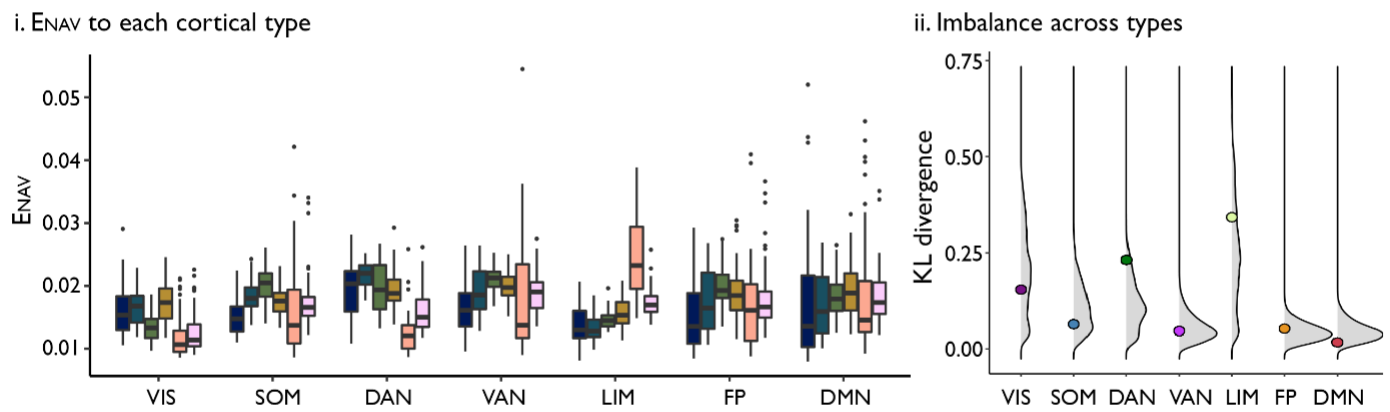

C | Balance of effective connectivity to cortical types i. Efferent connectivity to each cortical type
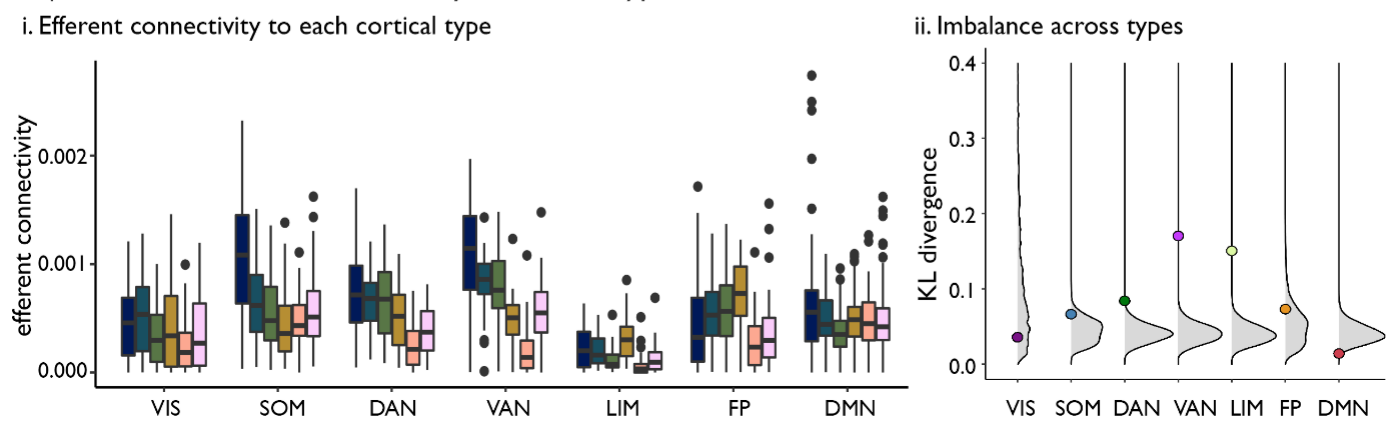

Figure 5: Uniqueness of the default mode network (DMN) relative to other functional networks. A) i. Crosstabulation of functional network by cortical type. Frequency is provided relative to total vertices in each type. VIS=visual. SOM=somatomotor. DAN=dorsal attention network. VAN=ventral attention network. LIM=limbic. FP=frontoparietal. DMN=default mode network. ii. Matrix depicts product-moment correlation ( $r$ ) of staining intensity profiles taken across the entire human brain. Distribution of $r$ within each network was compared to a null model using a Kullback-Leibler (KL) divergence test; shown in the lower right bar plots. The null model approximated a cytoarchitecturally homogenous network (normal probability function with mean=1 and $\mathrm{SD}=0.01$ ). B-C) i. Boxplots show median connectivity of each parcel to each cortical type stratified by functional network. ii. Imbalance of connectivity to distinct cortical types was evaluated as the KL divergence from a null model with equal Enav to each type. The coloured dots show the empirical values for each network and the grey density plots show the null distribution based on 10,000 spin permutations. 
We also stratified diffusion MRI derived navigation efficiency of each functional network with respect to cortical type (Figure 5Bi) and compared median navigation efficiency across cortical types to a balanced null model (i.e., equal median Enav across types). The DMN had the most balanced navigation efficiency across cortical types (Figure 5Bii, Supplementary Table 3), though the balance of the DMN was only marginally reduced compared to null models $\left(\mathrm{p}_{\mathrm{spin}}=0.11\right)$, which suggests that balanced navigation efficiency of the DMN is partly attributable to its size and spread. We repeated this analysis using efferent connectivity from rDCM, given the balance shown in Figure 4. Again, the DMN had the most balanced efferent connectivity across cortical types (Figure 5Ci, Supplementary Table 4), which was significant compared to spin permutations $\left(\mathrm{p}_{\text {spin }}=0.043\right)$.

\section{$\underline{\text { Robustness analyses }}$}

We confirmed the cytoarchitectural heterogeneity of the DMN by repeating our analyses with alternative atlases. The alternative atlases reflect the DMN as defined by consistency of deactivations during externally oriented tasks (Supplementary Figure 4) and a modified group-level atlas that uses a multi-session hierarchical Bayesian model to estimate individual-specific cortical networks [(Kong et al., 2019) Supplementary Figure 4]. In both cases, the DMN encompasses a broad range of cortical types. Additionally, using the Kong et al. (2019) atlas, we were able to ask whether cytoarchitectural heterogeneity of the DMN relate to previously described subnetworks, based on intrinsic functional connectivity. . We reformulated this question as a one-vs-one classification problem, using staining intensity profiles to predict the functional subdivisions (Supplementary Figure 5). Predictive accuracy was $42 \pm 7 \%, 74 \pm 3 \%$ and $73 \pm 4 \%$ for DMN-A, DMN-B and DMN-C, respectively. Given predictive accuracy exceeds chance levels, we can suggest that DMN subdivisions differ in their cytoarchitectural makeup, however, they are not clearly separable by cytoarchitecture, and each subdivision contains variable cytoarchitecture.

We examined the influence of subcortical structures and the hippocampus on the effective connectivity of the DMN in an extended rDCM (Supplementary Figure 6). Each structure was labelled as a new type and we repeated the analyses. While variance explained by cytoarchitectural features increased for both afferent $\left(\right.$ adj. $\left.\mathrm{R}^{2}=0.448, \mathrm{p}<0.001\right)$ and efferent connectivity $\left(\operatorname{adj} . \mathrm{R}^{2}=0.499, \mathrm{p}<0.001\right)$, the association of effective connectivity with cortical types was consistent with the original model. We observed mixed associations of afferent connectivity with E1 for the additional structures. Input from the caudate nucleus showed preference for flatter aspect of E1 $\left(\mathrm{r}=0.53, \mathrm{p}_{\text {spin }}<0.001\right)$, whereas the pallidum showed preference for the skewed aspect $\left(\mathrm{r}=-0.25, \mathrm{p}_{\text {spin }}=0.001\right)$. Additionally, while efferent connectivity of the DMN to the cortex was not clearly constrained by our definitions of cytoarchitecture, the flatter aspect of E1 exhibited stronger output to caudate, putamen, pallidum and nucleus accumbens $\left(0.31<\mathrm{r}<0.43\right.$, $\left.\mathrm{p}_{\text {spin }}<0.002\right)$.

\section{Replication analyses}

We repeated the imaging analyses in a separate dataset of 100 healthy young adults $(H C P)$. These analyses replicated our conclusions that (i) the DMN balances navigation efficiency across cortical types, (ii) navigation efficiency to the most granular types decreases along E1, (iii) input from non-DMN cortex to the DMN is focused on the skewed aspects of E1 and (iv) output of the DMN to non-DMN cortex is not clearly constrained by our cytoarchitectural definitions. Additionally, the DMN was unique in these features, relative to other functional networks. Key statistics are presented for both datasets in Supplementary Tables 3-5, and replication findings are presented in Supplementary Figures 7-9. 


\section{DISCUSSION}

Resolving the architecture of the DMN and its unique topographic position in the cortical landscape is central to understanding the neural basis of complex human behaviour. Combining post mortem histology with in vivo imaging, we discovered profound heterogeneity in the cytoarchitecture of the DMN, which supports its role in integrating diverse forms of information. We found that cytoarchitectural differentiation in the DMN aligns with its structural wiring and extrinsic information flow. These findings suggest a novel wiring diagram of structural and functional connectivity of the DMN that is compatible with its putative role in balancing internal and external information across cortical processing streams (Buckner et al., 2008; Yeshurun et al., 2021). Together, our analyses illustrate how the DMN protrudes from the sensory-fugal axis (Mesulam, 1998), while striking a balance in communication across this hierarchy.

Complementary theory- and data-driven analyses exposed the heterogeneous cytoarchitecture of the DMN. On the one hand, comparison of functional and cytoarchitectural atlases showed that the DMN harbours a wide range of cortical types, from eulaminate III to agranular. This type-based analysis demonstrates the extreme variation in cytoarchitecture of the DMN and that the DMN spans multiple steps of laminar elaboration (García-Cabezas et al., 2020; Von Economo and Koskinas, 1925; Von Economo and Triarhou, 2009). On the other hand, applying non-linear dimensionality reduction techniques to an ultra-high resolution histological reconstruction of a human brain, BigBrain (Amunts et al. 2013), highlighted an axis of cytoarchitectural differentiation within the DMN that is distinct to the gradient of laminar elaboration. Both the type-based and data-driven axes stretch between the primary sensory areas and the allocortex, but they capture different aspects of cytoarchitectural similarity in eulaminate II-I and dysgranular cortex. For instance, cortical types in the prefrontal cortex are related to neuronal density in layers II/III (Dombrowski, 2001), whereas the data-driven axis is related to the relative balance of staining in upper $v s$ lower layers also captured by intracortical profile skewness, as well as staining near the layer I/II boundary. In addition, cortical types are defined by topology, that is their spatial relations, whereas the data-driven axis is agnostic to space, which helps to reveal neurobiological complexity that exists within cortical areas (Amunts and Zilles, 2015).

The spatial pattern of cytoarchitecture differs between subregions of the DMN (Gadelmawla et al., 2002a). The parahippocampal region exhibits a medio-lateral cytoarchitectural gradient. This gradient resides within the previously described iso-to-allocortical transition of mesiotemporal lobe, which runs from the parahippocampal gyrus into the Cornu Ammonis subfields of the hippocampal formation (Braak and Braak, 1985; Paquola et al., 2020). Cytoarchitectural gradients were also observed in DMN subregions that include the cingulate, though deviations from the gradient were evident. Increasing distance from the limbus, here defined in line with (Pandya et al., 2015) as the edge of the cerebral hemispheres and the diencephalon,' was associated with more striations in cytoarchitecture. The striations morph into patches at greater distances from the limbus, as seen in the lateral frontal cortex. There the data-driven axis shows high frequency transitions in cytoarchitecture, which aligns with tract-tracing evidence of an interdigitated pattern of projections in prefrontal cortex (Goldman-Rakic and Schwartz, 1982; Selemon and GoldmanRakic, 1988), and recent resting-state fMRI work based on extended scanning sessions that show an interdigitated configuration of multiple functional networks, notably DMN subnetworks (Braga and Buckner, 2017). It has been proposed that interdigitation is a general organising principle of association circuits which serves to integrate disparate information (Goldman-Rakic and Schwartz, 1982). Thus, the differences in cytoarchitectural patterns across the cortex may be relevant to the cerebral mechanisms underlying information integration.

A core principle of neuroanatomy holds that topographies of cortical microstructure and connectivity are intrinsically related (Barbas, 1986; Goulas et al., 2019; Hilgetag and Grant, 2010; Pandya et al., 2015). In the DMN, we find a clear example of this relationship, in that the cytoarchitectural axis captures differences 
in structural and functional connectivity to other cortical territories. By fusing diffusion-based tractography with physical distance measurements into a model of navigation efficiency (Seguin et al., 2019, 2018), we found higher extrinsic connectivity of DMN subsections with more skewed profiles (i.e., lower E1), particularly to granular cortical types. Prior tract-tracing studies in macaques have focused upon circumscribed regions of the DMN, such as the precuneus/posterior cingulate, and have identified similar patterns of differential connectivity to primary sensory areas (Kobayashi and Amaral, 2003; Margulies et al., 2009). Our study shows the extension of this pattern across the DMN and indicates an interaction between the cytoarchitecture of both endpoints in determining navigation efficiency. The nature of this interaction suggests a novel principle of connectivity in association cortex. The efficiency of communication to primary sensory areas decreases along our data-driven cytoarchitectural axis, not according to cortical types. This somewhat contrasts with the structural model, which predicts connectivity based on similarity in cortical type. However, the structural model provides better explanation of longrange than short-range connectivity (Goulas et al., 2018) and long-range connections are prominent within the DMN (Betzel and Bassett, 2018). Thus, our data-driven axis and the gradient of laminar elaboration may capture patterns of extrinsic and intrinsic DMN connectivity, respectively.

Repeating the analysis with an rDCM of whole-brain effective connectivity, we observed decreasing afferent connectivity along the data-driven axis, though the effect was not constrained by the cortical type of the seed, as was evident with navigation efficiency. As such, we suggest that preferential navigation efficiency from skewed subunits of the DMN to more granular types may relate to the speed or directedness of communication, especially given more granular areas exhibit faster intrinsic timescales (Chaudhuri et al., 2015; Gao et al., 2020; Ito et al., 2020; Murray et al., 2014) and sensory areas require high fidelity information (Mesulam, 1998). In contrast, parcels of the DMN with flatter profiles (i.e. higher E1) are more insulated from primary sensory areas [also evident in (Margulies et al., 2016)] and receive less input from non-DMN cortex. This suggests that the characterisation of the DMN as distant from input (Murphy et al., 2018) is especially true for those insulated subsections of the DMN. The degree of insulation may be concordant with suppression during externally-oriented tasks, which is also regionally variable within the DMN (Ossandón et al., 2011). In line with our results, flatter subunits of the DMN, such as the medial prefrontal cortex, are suppressed for longer than those with more skewed profiles, such as the temporoparietal junction. Those insulated subunits of the DMN may play a more direct role in basal gangliathalamic feedback loops (Shine, 2021). We found that flatter DMN subunits had stronger output to the basal ganglia, which complements existing evidence on the predominance of cortico-striatal projections from the frontal cortex (Alexander et al., 1986; McFarland and Haber, 2000), as well as the patchiness of such projection sites (Lehéricy et al., 2004; Selemon and Goldman-Rakic, 1985). The connectivity analyses together illustrate the complementary functional roles of cytoarchitecturally distinct subunits of the DMN, from receivers on one-side of the cytoarchitectural axis to insulated, striatal projectors on the other side.

The combination of post mortem microscopy and in vivo imaging depends upon precise mapping between atlases. The present work leveraged a specialised multi-modal surface matching procedure (Lewis et al., 2020; Paquola et al., 2021), which minimises the misregistration error of landmarks to approximately $4 \mathrm{~mm}$, on par with standard in vivo registrations. The issue of precise mapping of functional networks to the BigBrain is further complicated by subject-specificity of functional network topographies (Braga and Buckner, 2017; Kong et al., 2019; Seitzman et al., 2019) and the impossibility of defining subject-specific functional networks on BigBrain. Nevertheless, our cytoarchitectural conclusions were supported when using a more conservative definition of the DMN, which was based on the consistency of DMN across individuals. The singular nature of BigBrain currently prohibits replication of the data-driven cytoarchitectural investigation and the examination of individual differences. Research on multiple post mortem brains, where every $15-20^{\text {th }}$ slice was processed, showed that the bauplan of cytoarchitectural areas 
is consistent across individuals, but the topography of areas differ (Amunts et al., 2020, 1999; Bludau et al., 2014). Further work on the individual variability of more detailed cytoarchitectural patterns is warranted. Here, we used parcel-based analysis of structural and functional connectivity, in order to focus on larger-scale patterns of cytoarchitecture that are more likely conserved across individuals.

Extant theories place the DMN as the apex of the sensory-fugal hierarchy (Margulies et al., 2016) or as a parallel network (Buckner and Krienen, 2013). Certain features of these theories are concordant with our results, such as (parts of) the DMN being insulated from input and the distinctiveness of information processing in the DMN. However, our analyses demonstrate that connectivity is organised along the most prominent cytoarchitectural axis of the DMN, which is not nested within or parallel to the sensory-fugal hierarchy. Instead, the DMN seems to protrude from the sensory-fugal hierarchy, with strong afferent connectivity on one end and insulation on the other. This architecture aligns with a rich club organisation of brain connectivity (van den Heuvel and Sporns, 2011), in which activation of a single rich club node through feeder connections can ignite meta-stable network dynamics (Gollo et al., 2015; Hong et al., 2019; Sepulcre et al., 2012). The areas with convergent afferents, as well as connections within the DMN, may enable recombinations that would not occur within sensory-fugal processing streams (Felleman and Van Essen, 1991). Such topological complexity is thought to be an important trade-off in development and evolution of biological neural networks (Bassett et al., 2010) and illustrates a distinctive role of the DMN in information integration.

This network-level conceptualisation has implications for how we think about the subdivisions of the DMN. Individualised assessments depict three domain specialised networks within the presently studied DMN map (Braga et al., 2020). The boundaries of these subnetworks do not likely conform to distinct cytoarchitectural types, as is also shown in our supplementary analysis on the group-level subdivisions (Andrews-Hanna et al., 2010; Yeo et al., 2011). Instead, we speculate that each domain specialised network comprises a diversity of cytoarchitecture and positions that support communication across the sensory-fugal hierarchy. The present work demonstrates how characterising the neuronal architecture of functional networks can enhance our understanding of network roles in complex behaviour. 


\section{METHODS}

\section{Histological data}

An ultra-high resolution Merker stained 3D volumetric histological reconstruction of a post mortem human brain from a 65-year-old male was obtained from the open-access BigBrain repository on September 1, 2020 [https://bigbrain.loris.ca/main.php; (Amunts et al., 2013)]. The post mortem brain was paraffin-

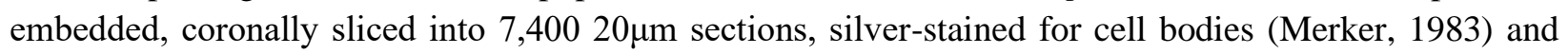
digitised. Manual inspection for artefacts (i.e., rips, tears, shears, and stain crystallisation) was followed by automatic repair procedures, involving non-linear alignment to a post mortem MRI, intensity normalisation, and block averaging (Lepage et al., 2010). The 3D reconstruction was implemented with a successive coarse-to-fine hierarchical procedure (Mohlberg et al., 2016). We downloaded the 3D volume at $100 \mu \mathrm{m}$ resolution, which was the highest resolution available for the whole brain. Computations were performed on inverted images, where staining intensity reflects greater cellular density and soma size. Geometric meshes approximating the outer and inner cortical interface (i.e., the GM/CSF boundary and the GM/WM boundary) with 163,842 matched vertices per hemisphere were also obtained (Lewis et al., 2014).

We constructed 50 equivolumetric surfaces between the outer and inner cortical surfaces (Wagstyl et al., 2018b). The equivolumetric model compensates for cortical folding by varying the Euclidean distance, $\rho$, between pairs of intracortical surfaces throughout the cortex to preserve the fractional volume between surfaces (Waehnert et al., 2014). $\rho$ was calculated as follows for each surface

$$
\rho=\frac{1}{A_{\text {out }}-A_{\text {in }}} \cdot\left(-A_{\text {in }}+\sqrt{\alpha A_{\text {out }}^{2}+(1-\alpha) A_{\text {in }}^{2}}\right)
$$

where $\alpha$ represents fraction of the total volume of the segment accounted for by the surface, while $\mathrm{A}_{\text {out }}$ and $\mathrm{A}_{\text {in }}$ represent the surface area of the outer and inner cortical surfaces, respectively. Vertex-wise staining intensity profiles were generated by sampling cell-staining intensities along linked vertices from the outer to the inner surface across the whole cortex. Smoothing was employed in tangential and axial directions to ameliorate the effects of artefacts, blood vessels, and individual neuronal arrangement (Wagstyl et al., 2018a). Smoothing across depths was enacted for each staining profile independently, using an iterative piece-wise linear procedure that minimises shrinkage [3 iterations, (Taubin, 1995)]. Additionally, surfacewise smoothing was performed at each depth independently and involved moving a 2-vertex FWHM Gaussian kernel across the surface mesh using SurfStat . The staining intensity profiles are made available in the BigBrainWarp toolbox [(https://github.com/caseypaquola/BigBrainWarp), (Paquola et al., 2021)].

\section{MRI acquisition - Primary analyses}

Primary MRI analyses were conducted on 40 healthy adults from the microstructure informed connectomics (MICs) cohort (14 females, mean \pm SD age $=30.4 \pm 6.7,2$ left-handed) (Royer et al., 2021). Scans were completed at the Brain Imaging Centre of the Montreal Neurological Institute and Hospital on a 3T Siemens Magnetom Prisma-Fit equipped with a 64-channel head coil. Two T1w scans with identical parameters were acquired with a 3D-MPRAGE sequence $(0.8 \mathrm{~mm}$ isotropic voxels, matrix $=320 \times 320,224$ sagittal slices, $\mathrm{TR}=2300 \mathrm{~ms}, \mathrm{TE}=3.14 \mathrm{~ms}, \mathrm{TI}=900 \mathrm{~ms}$, flip angle $=9^{\circ}, \mathrm{PAT}=2$, partial Fourier $=6 / 8$ ). Both $\mathrm{T} 1 \mathrm{w}$ scans were visually inspected to ensure minimal head motion before they were submitted to further processing. A spin-echo echo-planar imaging sequence with multi-band acceleration was used to obtain DWI data, consisting of three shells with b-values 300,700 , and $2000 \mathrm{~s} / \mathrm{mm}^{2}$ and 10,40 , and 90 diffusion weighting directions per shell, respectively $\left(\mathrm{TR}=3500 \mathrm{~ms}, \mathrm{TE}=64.40 \mathrm{~ms}, 1.6 \mathrm{~mm}\right.$ isotropic voxels, flip angle $=90^{\circ}$, 
refocusing flip angle $=180^{\circ}, \mathrm{FOV}=224 \times 224 \mathrm{~mm}^{2}$, slice thickness $=1.6 \mathrm{~mm}$, multiband factor $=3$, echo spacing $=0.76 \mathrm{~ms}$, number of b0 images $=3$ ). One $7 \mathrm{~min}$ rs-fMRI scan was acquired using multiband accelerated 2D-BOLD echo-planar imaging $\left(\mathrm{TR}=600 \mathrm{~ms}, \mathrm{TE}=30 \mathrm{~ms}, 3 \mathrm{~mm}\right.$ isotropic voxels, flip angle $=52^{\circ}$, FOV $=240 \times 240 \mathrm{~mm}^{2}$, slice thickness $=3 \mathrm{~mm}, \mathrm{mb}$ factor $=6$, echo spacing $=0.54 \mathrm{~ms}$ ). Participants were instructed to keep their eyes open, look at a fixation cross, and not fall asleep. Two spin-echo images with reverse phase encoding were also acquired for distortion correction of the rs-fMRI scans (phase encoding $=\mathrm{AP} / \mathrm{PA}, 3 \mathrm{~mm}$ isotropic voxels, $\mathrm{FOV}=240 \times 240 \mathrm{~mm}^{2}$, slice thickness $=3 \mathrm{~mm}, \mathrm{TR}=4029 \mathrm{~ms}$, $\mathrm{TE}=48 \mathrm{~ms}$, flip angle $=90^{\circ}$, echo spacing $=0.54 \mathrm{~ms}$, bandwidth $=2084 \mathrm{~Hz} / \mathrm{Px}$ ).

Each T1w scan was deobliqued and reoriented. Both scans were then linearly co-registered and averaged, automatically corrected for intensity nonuniformity (Tustison et al., 2010), and intensity normalized. Resulting images were skull-stripped, and non-isocortical structures were segmented using FSL FIRST (Jenkinson et al., 2012). Different tissue types (cortical and subcortical grey matter, white matter, cerebrospinal fluid) were segmented to perform anatomically constrained tractography (Smith et al., 2012). Cortical surface segmentations were generated from native T1w scans using FreeSurfer 6.0 (Dale et al., 1999; Fischl et al., 1999b, 1999a).

DWI data were pre-processed using MRtrix (Tournier et al., 2019, 2012). DWI data underwent b0 intensity normalization, and were corrected for susceptibility distortion, head motion, and eddy currents. Required anatomical features for tractography processing (e.g., tissue type segmentations, parcellations) were nonlinearly co-registered to native DWI space using the deformable SyN approach implemented in Advanced Neuroimaging Tools (ANTs) (Avants et al., 2008; Tustison and Avants, 2013). Diffusion processing and tractography were performed in native DWI space. We performed anatomically-constrained tractography using tissue types segmented from each participant's pre-processed T1w images registered to native DWI space (Smith et al., 2012). We estimated multi-shell and multi-tissue response functions (Christiaens et al., 2015) and performed constrained spherical-deconvolution and intensity normalization (Jeurissen et al., 2014). We initiated the tractogram with 40 million streamlines (maximum tract length=250; fractional anisotropy cutoff=0.06). We applied spherical deconvolution informed filtering of tractograms (SIFT2) to reconstruct whole brain streamlines weighted by cross-sectional multipliers (Smith et al., 2015). The reconstructed cross-section streamlines were averaged within 400 spatially contiguous, functionally defined parcels (Schaefer et al., 2018), also warped to DWI space.

rs-fMRI images were pre-processed using AFNI (Cox, 1996) and FSL (Jenkinson et al., 2012). The first five volumes were discarded to ensure magnetic field saturation. Images were reoriented, and motion as well as distortion corrected. Nuisance variable signal was removed using an ICA-FIX classifier (SalimiKhorshidi et al., 2014) and by performing spike regression. Native timeseries were mapped to individual surface models using a boundary-based registration (Greve and Fischl, 2009) and smoothed using a Gaussian kernel (FWHM=10mm, smoothing performed on native midsurface mesh) using workbench (Glasser et al., 2013). For isocortical regions, timeseries were sampled on native surfaces and averaged within 400 spatially contiguous, functionally defined parcels (Schaefer et al., 2018). For non-isocortical regions, timeseries were averaged within native parcels of the nucleus accumbens, amygdala, caudate nucleus, hippocampus, pallidum, putamen, and thalamus (Jenkinson et al., 2012). 


\section{MRI acquisition - Replication analyses}

Replication MRI analyses were conducted in 100 unrelated healthy adults ( 66 females, mean \pm SD age $=28.8 \pm 3.8$ years) from the minimally preprocessed S900 release of the Human Connectome Project (HCP) (Glasser et al., 2013). MRI data were acquired on the HCP's custom 3T Siemens Skyra equipped with a 32-channel head coil. Two T1w images with identical parameters were acquired using a 3DMPRAGE sequence $(0.7 \mathrm{~mm}$ isotropic voxels, matrix $=320 \times 320,256$ sagittal slices; TR=2400ms, $\mathrm{TE}=2.14 \mathrm{~ms}$, $\mathrm{TI}=1000 \mathrm{~ms}$, flip angle $=8^{\circ} ; \mathrm{PAT}=2$ ). Two $\mathrm{T} 2 \mathrm{w}$ images were acquired using a 3D T2-SPACE sequence with identical geometry $(\mathrm{TR}=3200 \mathrm{~ms}, \mathrm{TE}=565 \mathrm{~ms}$, variable flip angle, $\mathrm{iPAT}=2)$. A spin-echo EPI sequence was used to obtain diffusion weighted images, consisting of three shells with $b$-values 1000, 2000, and $3000 \mathrm{~s} / \mathrm{mm}^{2}$ and up to 90 diffusion weighting directions per shell $(\mathrm{TR}=5520 \mathrm{~ms}, \mathrm{TE}=89.5 \mathrm{~ms}$, flip angle $=78^{\circ}$, refocusing flip angle $=160^{\circ}, \mathrm{FOV}=210 \times 180$, matrix $=178 \times 144$, slice thickness $=1.25 \mathrm{~mm}, \mathrm{mb}$ factor $=3$, echo spacing $=0.78 \mathrm{~ms}$ ). Four rs-fMRI scans were acquired using multi-band accelerated 2DBOLD echo-planar imaging $(2 \mathrm{~mm}$ isotropic voxels, matrix $=104 \times 90,72$ sagittal slices, $\mathrm{TR}=720 \mathrm{~ms}$, $\mathrm{TE}=33 \mathrm{~ms}$, flip angle $=52^{\circ}, \mathrm{mb}$ factor $=8,1200$ volumes $/ \mathrm{scan}, 3456$ seconds). Only the first session was investigated in the present study. Participants were instructed to keep their eyes open, look at a fixation cross, and not fall asleep. Nevertheless, some subjects were drowsy and may have fallen asleep (Glasser et al., 2018), and the group-averages investigated in the present study do not address these inter-individual differences. MRI data underwent HCP's minimal preprocessing (Glasser et al., 2013). Cortical surface models were constructed using Freesurfer 5.3-HCP (Dale et al., 1999; Fischl et al., 1999a, 1999b), with minor modifications to incorporate both T1w and T2w (Glasser and Van Essen, 2011).

Diffusion MRI data underwent correction for geometric distortions and head motion (Glasser et al., 2013). Tractographic analysis was based on MRtrix3 (https://www.mrtrix.org). Response functions for each tissue type were estimated using the dhollander algorithm (Dhollander et al., 2016). Fibre orientation distributions (i.e., the apparent density of fibres as a function of orientation) were modelled from the diffusion-weighted MRI with multi-shell multi-tissue spherical deconvolution (Jeurissen et al., 2014), then values were normalised in the log domain to optimise the sum of all tissue compartments towards 1 , under constraints of spatial smoothness. Anatomically constrained tractography was performed systematically by generating streamlines using second order integration over fibre orientation distributions with dynamic seeding (Smith et al., 2015; Tournier et al., 2007). Streamline generation was aborted when 40 million streamlines had been accepted. Using a spherical-deconvolution informed filtering of tractograms (SIFT2) approach, interregional tract strength (TS) was taken as the streamline count weighted by the estimated cross section (Smith et al., 2015). The reconstructed cross-section streamlines were averaged within 400 spatially contiguous, functionally defined parcels (Schaefer et al., 2018), also warped to DWI space.

BOLD timeseries were corrected for gradient nonlinearity, head motion, bias field and scanner drifts, then structured noise components were removed using ICA-FIX, further reducing the influence of motion, nonneuronal physiology, scanner artefacts and other nuisance sources (Salimi-Khorshidi et al., 2014). The rsfMRI data were resampled from volume to MSMAll functionally aligned surface space (Robinson et al., 2018 , 2014) and averaged within 400 spatially contiguous, functionally defined parcels (Schaefer et al., 2018). 


\section{Comparison of cortical maps}

Functional networks were defined using the widely used Yeo et al., (2011) atlas. The atlas reflects clustering of cortical vertices according to similarity in resting state functional connectivity profiles, acquired in 1000 healthy young adults. Cortical types were assigned to Von Economo areas (Scholtens et al., 2016; Von Economo and Koskinas, 1925), based on a recent re-analysis of Von Economo micrographs (GarcíaCabezas et al., 2020). Several features were used to identify the type, including "development of layer IV, prominence (denser cellularity and larger neurons) of deep (V-VI) or superficial (II-III) layers, definition of sublayers (e.g., IIIa and IIIb), sharpness of boundaries between layers, and presence of large pyramids in superficial layers" (García-Cabezas et al., 2020). Cortical types synopsise degree of granularity, from high laminar elaboration in koniocortical areas, six identifiable layers in eulaminate III-I, poorly differentiated layers in dysgranular and absent layers in agranular.

The proportion of DMN vertices assigned to each cortical type was calculated on a common surface template, fsaverage5 (Dale et al., 1999). Throughout the statistical analyses, we employ a spin permutation framework to compute p-values and evaluate significance. This procedure involved generating a null distribution by rotating one brain map 10,000 times and recomputing the outcome of interest. Then, we

calculate $\quad p_{\text {spin }}=1-\frac{\Sigma(\text { empirical }>\text { permutations })}{\text { totalpermutations }} \quad$ and/or $\quad p_{\text {spin }}=1-\frac{\Sigma(\text { empirical }<\text { permutations })}{\text { total permutations }}$ (Alexander-Bloch et al., 2018; Vos de Wael et al., 2020). Crucially, the null distribution preserves the spatial structure of both brain maps, which establishes the plausibility of a random alignment of the maps explaining their correspondence. Generally, we deemed significance $\mathrm{p}<0.05$ for one-tailed tests and $\mathrm{p}<0.025$ for two-tailed tests. Additionally, we used Bonferroni correction when multiple univariate comparisons were made using the same response variable.

\section{Data-driven cytoarchitectural axis within the DMN}

The functional network atlases were transformed to the BigBrain surface using a specially optimised multimodal surface matching algorithm (Lewis et al., 2020). The pattern of cytoarchitectural heterogeneity in the DMN was revealed using non-linear manifold learning. The approach involved calculating pair-wise product-moment correlations of BigBrain staining intensity profiles, controlling for the average DMN staining intensity profile. Negative values were zeroed to emphasise the non-shared similarities. Diffusion map embedding of the correlation matrix was employed to gain a low dimensional representation of cytoarchitectural patterns (Coifman and Lafon, 2006; Margulies et al., 2016). Diffusion map embedding belongs to the family of graph Laplacians, which involve constructing a reversible Markov chain on an affinity matrix. Compared to other nonlinear manifold learning techniques, the algorithm is relatively robust to noise and computationally inexpensive (Tenenbaum et al., 2000; Von Luxburg, 2007). A single parameter $\alpha$ controls the influence of the sampling density on the manifold ( $\alpha=0$, maximal influence; $\alpha=$ 1, no influence). As in previous studies (Margulies et al., 2016; Vos de Wael et al., 2018), we set $\alpha=0.5$, a choice retaining the global relations between data points in the embedded space. Notably, different alpha parameters had little to no impact on the first two eigenvectors (spatial correlation of eigenvectors, $r>0.99$ ).

The DMN comprises 71,576 vertices on the BigBrain surface, each associated with approximately $1 \mathrm{~mm}^{2}$ of surface area. Pair-wise correlation and manifold learning on 71,576 data points was computationally infeasible, however. Thus, we performed a 6-fold mesh decimation on the BigBrain surface to select a subset of vertices that preserve the overall shape of the mesh (The MathWorks, 2019). Then, we assigned each non-selected vertex to the nearest maintained vertex, determined by shortest path on the mesh (ties were solved by shortest Euclidean distance). Staining intensity profiles were averaged within each surface 
patch of the DMN, then the dimensionality reduction procedure was employed. Subsequent analyses focused on the first eigenvector (E1), which explained the most variance in the affinity matrix.

Local variations in E1 were examined within spatially contiguous regions of the DMN, which were defined using SurfStat (Worsley et al., 2009). Quantitative description of E1 heterogeneity within each subregion was achieved by adopting an approach from microscopic materials physics for characterising the roughness of a surface (Gadelmawla et al., 2002b). Research in landscape ecology demonstrates the benefits of using roughness parameters on larger-scale domains, especially where features vary continuously (McGarigal et al., 2009). Given the continuity of neurobiological attributes (Fukuchi-Shimogori and Grove, 2001; Fulcher et al., 2019), roughness parameters are well suited to characterise subregion patterns of E1. Roughness parameters may be categorised as amplitude, spatial or hybrid measures. Amplitude parameters relate to the vertical characteristics of the surface deviations, spatial to the horizontal characteristics and hybrid to the combination. In the present work, the surface mesh was used to define neighbourhoods and spatial relations. A subset of parameters from Gadelmawla et al. (2002b) were adopted (the full list is provided in Supplementary Table 1). Certain parameters were excluded if requirements for the equation could not assumed [i.e., more than 3 peaks or troughs within a region $\left(\mathrm{R}_{\mathrm{z}}, \mathrm{R}_{\mathrm{ti}}, \mathrm{R}_{\mathrm{tm}}, \mathrm{R}_{\mathrm{y}}, \mathrm{R}_{3 \mathrm{y}}, \mathrm{R}_{3 \mathrm{z}}\right)$ ], the equation requires a priori segmentation of the surface $\left(1_{0}\right)$, the equation requires an a priori threshold level (high spot count, $\mathrm{H}_{\mathrm{u}}, \mathrm{H}_{\mathrm{s}}, \mathrm{P}_{\mathrm{u}}, \mathrm{P}_{\mathrm{tp}}$ ) or the equation could not be directly translated from 2D to 3D (power spectral density, autocorrelation function, amplitude density function, number of inflection points). In total, the present work includes 9 amplitude, 5 spatial and 7 hybrid parameters. Following calculation of the roughness parameters, the coefficient of variation was calculated across subregions to determine which parameters distinguish subregions.

The relation of E1 to staining intensity profiles was visually inspected by discretising E1 into 100 bins, averaging profiles within bins and plotting the profiles with colours matched to bin-average E1. Specific cytoarchitectural features that vary across E1 were identified from a set of 54 [50 depth-wise intensities and 4 central moments (Paquola et al., 2019a)]. The features were z-standardised and $70 \%$ of observations were used to train a random forest regression. Training involved a grid search for optimal hyperparameters (maximum depth and number of estimators) and feature selection. Feature importance was estimated as the mean decrease in model accuracy with feature weightings shuffled and features with above average importance were selected. Note, feature importance values must be taken in the context of the model, and they represent the relative variance explained within the model. Both training procedures used five-fold cross validation and mean squared error as a measure of model accuracy. The accuracy (mean squared error) and explanatory power $\left(\mathrm{R}^{2}\right)$ of the selected model were evaluated on the remaining $30 \%$ of observations (test set).

\section{Structural connectivity of the DMN}

Extrinsic connectivity of DMN subunits was mapped using structural connectomes, derived from diffusionbased tractography. Edge weights of the structural connectomes, representing number of streamlines, were remapped to edge lengths using a log-based transformation: $\mathrm{L}=-\log 10(\mathrm{~W} /(\max (\mathrm{W})+\min (\mathrm{W}>0))$. This log-based transformation attenuates extreme weights and ensures the maximum edge weight is mapped to a positive value. Euclidean distance was calculated between the centroid coordinate of each parcel. Communication in the structural connectome was modelled using navigation (Seguin et al., 2018), also known as greedy routing (Muscoloni et al., 2017). Navigation combines the structural connectome with physical distances, providing a routing strategy that recapitulates invasive, tract-tracing measures of communication (Seguin et al., 2019). In brief, navigation involves step-wise progression from node i to node $\mathrm{j}$, where each step is determined by spatial proximity to $\mathrm{j}$. Navigation is the sum distances of the 
selected path and navigation efficiency $\left(\mathrm{E}_{\mathrm{nav}}\right)$ its inverse; providing an intuitive metric of communication efficiency between two regions. Navigation efficiency was calculated within each hemisphere separately.

Each parcel was labelled according to functional network, modal cortical type and, if part of the DMN, average E1 value. Parcel-average E1 values were calculated by transforming the parcellation scheme to the BigBrain surface and averaging within parcel (Lewis et al., 2020). We selected DMN rows and non-DMN columns of the $\mathrm{E}_{\text {nav }}$ matrix and stratified the non-DMN columns by cortical type. First, we compared typespecific $\mathrm{E}_{\text {nav }}$ against spin permutations of the cortical type atlas to test for hyper- or hypo-navigation between the DMN and any specific type. Then, for each cortical type, we calculated row-wise median of $\mathrm{E}_{\mathrm{nav}}$, concatenated the hemispheres and evaluated the product-moment correlation of row-wise median $\mathrm{E}_{\text {nav }}$ with E1. The two-tailed spin permutations p-value was corrected for six comparisons, meaning we deemed significance where $\mathrm{p}<0.004$. The analysis indicates whether communication efficiency of the DMN to a specific cortical type varies along the data-driven cytoarchitectural axis. We also repeated the analysis with $\mathrm{DMN} \times$ whole cortex matrix and a DMN $\times$ DMN matrix to test whether the cytoarchitectural variations played a similar role for extrinsic and intrinsic communication of the DMN.

\section{Effective connectivity of the DMN}

The position of the DMN in large-scale cortical dynamics was explored with regression dynamic causal modelling [rDCM;(Frässle et al., 2017)], freely available as part of the TAPAS software package (Frässle et al., 2021a), a highly scalable generative model of effective connectivity that allows inferences on the directionality of signal flow. Input to and output of the DMN were evaluated separately in the following analyses. First, we estimated the variance explained in DMN effective connectivity by cortical types and the data-driven cytoarchitectural axis. To do so, we calculated the type-specific effective connectivity for each parcel of the DMN, that is the average effective connectivity to non-DMN parcels of a certain cortical type. Then, we fit a multiple linear regression model with type-specific effective connectivity as the response variable, cortical type as a categorical predictor and E1 as a continuous predictor. We conducted post-hoc analyses using spin permutations to determine the specific influences of cortical type and E1. Specifically, we compared (i) type-specific effectivity connectivity of the entire DMN against spin permutations of the cortical type atlas, (ii) correlation of E1 with average non-DMN effective connectivity against spin permutations of E1, and (iii) correlation of E1 with type-specific effective connectivity of the entire DMN against spin permutations of E1. These tests represent the cortical type effect, E1 effect and their interaction, respectively.

The rDCM was re-generated with additional non-isocortical regions, specifically the nucleus accumbens, amygdala, caudate nucleus, hippocampus, pallidum, putamen, and thalamus. The above analyses were repeated for the isocortex to assess robustness of the model. Additionally, correlation of E1 with effective connectivity was calculated and compared against spin permutations of E1 for each non-isocortical region. The correlation values were visualised on non-isocortical regions using the ENIGMA toolbox (Paquola et al., 2021).

\section{$\underline{\text { Robustness to atlas definition }}$}

The robustness of cytoarchitectural heterogeneity to the DMN definition was assessed with two alternative atlases. Given the origins of the DMN as a "task-negative" set of regions (Raichle et al., 2001; Shulman et al., 1997), the first alternative atlas involved identifying regions that are consistently deactivated during externally-oriented tasks. In line with a recent review (Smallwood et al., 2021b), we used pre-defined contrast maps from 787 healthy young adults of the Human Connectome Project 
(“HCP_S900_GroupAvg_v1 Dataset”). Each map represents the contrast between BOLD response during a task and at baseline. Fifteen tasks were selected [Working Memory (WM) - 2 Back, WM-0 Back, WMBODY, WM-FACE, WM-PLACE, WM-TOOL, GAMBLING-PUNISH, GAMBLING-REWARD, MOTOR-AVG, SOCIAL-RANDOM, SOCIAL - Theory of Mind (TOM), RELATIONAL-MATCH, RELATIONAL-REL, EMOTION-FACES, EMOTION-SHAPES] to correspond to initial studies of the DMN (Raichle et al., 2001). Task-related deactivation was defined at each vertex and for each contrast as z-score $\leq-5$, which is consistent with contemporary statistical thresholds used in neuroimaging to reduce false positives (Eklund et al., 2016). A probabilistic atlas of the DMN was calculated as the percentage of contrasts with task-related deactivation. The second alternative atlas represents the probability of the DMN at each vertex, calculated across 1029 individual-specific functional network delineations (Kong et al., 2019). For each alternative atlas, we calculated the proportions of cortical types across a range of probabilistic thresholds (5-95\%, at 5\% increments) to determine whether the discovered cytoarchitectural heterogeneity of the DMN was robust to atlas definition.

Discrimination of functional sub-networks by cytoarchitectural eigenvectors was formulated as a classification problem. DMN vertices were reassigned to the one of three subnetworks based on a previously published atlas (Yeo et al., 2011). Four folds of equal size and equal sub-network proportions were collated, meaning the number of vertices from each sub-network in each fold was capped based on the size of the smallest network. Vertex-wise values of the top 10 cytoarchitectural eigenvectors were used to predict functional sub-network with a one-vs-one linear support vector classifier (Pedregosa et al., 2011). Within each fold, $70 \%$ of vertices were used for training and 30\% for testing. The contribution of each eigenvector was estimated by comparing the full model accuracy to leave-one-eigenvector-out model accuracy.

\section{Uniqueness of the DMN relative to other functional networks}

To determine the unique features of the DMN, relative to other functional networks, we compared cortical type assignments, cytoarchitectural heterogeneity and connectivity with cortical types to other canonical intrinsic functional networks (i.e., visual, somatomotor, dorsal attention, ventral attention, limbic and frontoparietal) (Yeo et al., 2011). Chi-squared tests measured the inequality of the functional network membership within each cortical type. Cytoarchitectural heterogeneity was further evaluated by calculating product-moment correlation of staining intensity profiles within each network. The Kullback-Leibler divergence of the empirical distribution of correlation values was calculated relative to a null model that represented a homogenous network. The homogenous network was modelled as a normal distribution with mean 1 and standard deviation 0.01 , sampled from 0.9 to 1 in 0.01 increments. Finally, given the lack of type-preference in $E_{\text {nav }}$ and efferent connectivity of the DMN, we estimated the imbalance in $E_{\text {nav }}$ and efferent connectivity across cortical types for each functional network. Imbalanced was calculated as the Kullback-Leibler divergence from a null model with equal median connectivity to each type and compared to spin permutations of the functional network atlas. 


\section{References}

Alcalá-López, D., Smallwood, J., Jefferies, E., Van Overwalle, F., Vogeley, K., Mars, R.B., Turetsky, B.I., Laird, A.R., Fox, P.T., Eickhoff, S.B., Bzdok, D., 2018. Computing the social brain connectome across systems and states. Cereb. Cortex 28, 2207-2232. https://doi.org/10.1093/cercor/bhx121

Alexander-Bloch, A.F., Shou, H., Liu, S., Satterthwaite, T.D., Glahn, D.C., Shinohara, R.T., Vandekar, S.N., Raznahan, A., 2018. On testing for spatial correspondence between maps of human brain structure and function. Neuroimage 178, 540-551. https://doi.org/10.1016/j.neuroimage.2018.05.070

Alexander, G.E., DeLong, M.R., Strick, P.L., 1986. Parallel organization of functionally segregated circuits linking basal ganglia and cortex. Annu. Rev. Neurosci. VOL. 9, 357-381. https://doi.org/10.1146/annurev.ne.09.030186.002041

Alves, P.N., Foulon, C., Karolis, V., Bzdok, D., Margulies, D.S., Volle, E., Thiebaut de Schotten, M., 2019. An improved neuroanatomical model of the default-mode network reconciles previous neuroimaging and neuropathological findings. Commun. Biol. 2. https://doi.org/10.1038/s42003019-0611-3

Amunts, K., Lepage, C., Borgeat, L., Mohlberg, H., Dickscheid, T., Rousseau, M.-E., Bludau, S., Bazin, P.-L., Lewis, L.B., Oros-Peusquens, A.-M., Shah, N.J., Lippert, T., Zilles, K., Evans, A.C., 2013. BigBrain: An Ultrahigh-Resolution 3D Human Brain Model. Science (80-. ). 340, 1472-1475. https://doi.org/10.1126/science.1235381

Amunts, K., Mohlberg, H., Bludau, S., Zilles, K., 2020. Julich-Brain: A 3D probabilistic atlas of the human brain's cytoarchitecture. Science (80-. ). 4588, 1-10.

https://doi.org/10.1126/science.abb4588

Amunts, K., Schleicher, A., Bürgel, U., Mohlberg, H., Uylings, H.B.M., Zilles, K., 1999. Broca's region revisited: Cytoarchitecture and intersubject variability. J. Comp. Neurol. 412, 319-341. https://doi.org/10.1002/(SICI)1096-9861(19990920)412:2<319::AID-CNE10>3.0.CO;2-7

Amunts, K., Zilles, K., 2015. Architectonic Mapping of the Human Brain beyond Brodmann. Neuron. https://doi.org/10.1016/j.neuron.2015.12.001

Anderson, J.S., Ferguson, M.A., Lopez-Larson, M., Yurgelun-Todd, D., 2011. Connectivity Gradients Between the Default Mode and Attention Control Networks. Brain Connect. 1, 147-157. https://doi.org/10.1089/brain.2011.0007

Andrews-Hanna, J.R., Reidler, J.S., Sepulcre, J., Poulin, R., Buckner, R.L., 2010. Functional-Anatomic Fractionation of the Brain's Default Network. Neuron 65, 550-562. https://doi.org/10.1016/j.neuron.2010.02.005

Avants, B.B., Epstein, C.L., Grossman, M., Gee, J.C., 2008. Symmetric diffeomorphic image registration with cross-correlation: Evaluating automated labeling of elderly and neurodegenerative brain. Med. Image Anal. 12, 26-41. https://doi.org/10.1016/j.media.2007.06.004

Barbas, H., 2015. General Cortical and Special Prefrontal Connections: Principles from Structure to Function. Annu. Rev. Neurosci. 38, 269-289. https://doi.org/10.1146/annurev-neuro-071714033936

Barbas, H., 1986. Pattern in the laminar origin of corticocortical connections. J. Comp. Neurol. 252, 415422. https://doi.org/10.1002/cne.902520310 
Barbas, H., Rempel-Clower, N., 1997. Cortical structure predicts the pattern of corticocortical connections. Cereb. Cortex 7, 635-646. https://doi.org/10.1093/cercor/7.7.635

Bassett, D.S., Greenfield, D.L., Meyer-Lindenberg, A., Weinberger, D.R., Moore, S.W., Bullmore, E.T., 2010. Efficient physical embedding of topologically complex information processing networks in brains and computer circuits. PLoS Comput. Biol. 6, e1000748.

https://doi.org/10.1371/journal.pcbi.1000748

Betzel, R.F., Bassett, D.S., 2018. Specificity and robustness of long-distance connections in weighted, interareal connectomes. Proc. Natl. Acad. Sci. U. S. A. 115, E4880-E4889. https://doi.org/10.1073/pnas.1720186115

Bludau, S., Eickhoff, S.B., Mohlberg, H., Caspers, S., Laird, A.R., Fox, P.T., Schleicher, A., Zilles, K., Amunts, K., 2014. Cytoarchitecture, probability maps and functions of the human frontal pole. Neuroimage 93, 260-275. https://doi.org/10.1016/j.neuroimage.2013.05.052

Braak, H., Braak, E., 1985. On areas of transition between entorhinal allocortex and temporal isocortex in the human brain. Normal morphology and lamina-specific pathology in Alzheimer's disease. Acta Neuropathol. 68, 325-332. https://doi.org/10.1007/BF00690836

Braga, R.M., Buckner, R.L., 2017. Parallel Interdigitated Distributed Networks within the Individual Estimated by Intrinsic Functional Connectivity. Neuron 95, 457-471.e5. https://doi.org/10.1016/j.neuron.2017.06.038

Braga, R.M., DiNicola, L.M., Becker, H.C., Buckner, R.L., 2020. Situating the left-lateralized language network in the broader organization of multiple specialized large-scale distributed networks. J. Neurophysiol. 124, 1415-1448. https://doi.org/10.1152/JN.00753.2019

Braga, R.M., Sharp, D.J., Leeson, C., Wise, R.J.S., Leech, R., 2013. Echoes of the brain within default mode, association, and heteromodal cortices. J. Neurosci. 33, 14031-9. https://doi.org/10.1523/JNEUROSCI.0570-13.2013

Brandman, T., Malach, R., Simony, E., 2021. The surprising role of the default mode network in naturalistic perception. Commun. Biol. 4, 1-9. https://doi.org/10.1038/s42003-020-01602-z

Brodmann, K., 1903. Beitrage zur histologischen Lokalisation der Grosshirnrinde. Die Regio Rolandica. J. für Psychol. und Neurol. 2, 79-107.

Buckner, R.L., Andrews-Hanna, J.R., Schacter, D.L., 2008. The brain's default network: Anatomy, function, and relevance to disease. Ann. N. Y. Acad. Sci. 1124, 1-38. https://doi.org/10.1196/annals.1440.011

Buckner, R.L., Krienen, F.M., 2013. The evolution of distributed association networks in the human brain. Trends Cogn. Sci. https://doi.org/10.1016/j.tics.2013.09.017

Caspers, S., Eickhoff, S.B., Geyer, S., Scheperjans, F., Mohlberg, H., Zilles, K., Amunts, K., 2008. The human inferior parietal lobule in stereotaxic space. Brain Struct. Funct. 212, 481-495. https://doi.org/10.1007/s00429-008-0195-z

Caspers, S., Geyer, S., Schleicher, A., Mohlberg, H., Amunts, K., Zilles, K., 2006. The human inferior parietal cortex: Cytoarchitectonic parcellation and interindividual variability. Neuroimage 33, 430448. https://doi.org/10.1016/j.neuroimage.2006.06.054

Chaudhuri, R., Knoblauch, K., Gariel, M.-A., Kennedy, H., Wang, X.-J., 2015. A Large-Scale Circuit Mechanism for Hierarchical Dynamical Processing in the Primate Cortex. Neuron 88, 419-431. https://doi.org/10.1016/J.NEURON.2015.09.008 
Christiaens, D., Reisert, M., Dhollander, T., Sunaert, S., Suetens, P., Maes, F., 2015. Global tractography of multi-shell diffusion-weighted imaging data using a multi-tissue model. Neuroimage 123, 89101.

Cisek, P., 2012. Making decisions through a distributed consensus. Curr. Opin. Neurobiol. https://doi.org/10.1016/j.conb.2012.05.007

Coifman, R.R., Lafon, S., 2006. Diffusion maps. Appl. Comput. Harmon. Anal. 21, 5-30. https://doi.org/10.1016/j.acha.2006.04.006

Coifman, R.R., Lafon, S., Lee, A.B., Maggioni, M., Nadler, B., Warner, F., Zucker, S.W., 2005. Geometric diffusions as a tool for harmonic analysis and structure definition of data: Multiscale methods. Proc. Natl. Acad. Sci. 102, 7432-7437. https://doi.org/10.1073/pnas.0500896102

Cox, R.W., 1996. AFNI: software for analysis and visualization of functional magnetic resonance neuroimages. Comput. Biomed. Res. 29, 162-173.

Dale, A.M., Fischl, B., Sereno, M.I., 1999. Cortical surface-based analysis. I. Segmentation and surface reconstruction. Neuroimage 9, 179-194. https://doi.org/10.1006/nimg.1998.0395

Davey, C.G., Pujol, J., Harrison, B.J., 2016. Mapping the self in the brain's default mode network. Neuroimage 132, 390-397. https://doi.org/10.1016/j.neuroimage.2016.02.022

Dhollander, T., Raffelt, D., Connelly, A., 2016. Unsupervised 3-tissue response function estimation from single-shell or multi-shell diffusion MR data without a co-registered T1 image, in: ISMRM Workshop on Breaking the Barriers of Diffusion MRI. p. 5.

Dixon, M.L., Andrews-Hanna, J.R., Spreng, R.N., Irving, Z.C., Mills, C., Girn, M., Christoff, K., 2017. Interactions between the default network and dorsal attention network vary across default subsystems, time, and cognitive states. Neuroimage 147, 632-649. https://doi.org/10.1016/j.neuroimage.2016.12.073

Dombrowski, S.M., 2001. Quantitative Architecture Distinguishes Prefrontal Cortical Systems in the Rhesus Monkey. Cereb. Cortex 11, 975-988. https://doi.org/10.1093/cercor/11.10.975

Eklund, A., Nichols, T.E., Knutsson, H., 2016. Cluster failure: Why fMRI inferences for spatial extent have inflated false-positive rates. Proc Natl Acad Sci U S A 113, 7900-7905. https://doi.org/10.1073/pnas.1602413113

Felleman, D.J., Van Essen, D.C., 1991. Distributed hierarchical processing in the primate cerebral cortex. Cereb. Cortex 1, 1-47. https://doi.org/10.1093/cercor/1.1.1-a

Figley, T.D., Bhullar, N., Courtney, S.M., Figley, C.R., 2015. Probabilistic atlases of default mode, executive control and salience network white matter tracts: An fMRI-guided diffusion tensor imaging and tractography study. Front. Hum. Neurosci. 9, 585.

https://doi.org/10.3389/fnhum.2015.00585

Fischl, B., Sereno, M.I., Dale, A.M., 1999a. Cortical surface-based analysis. II: Inflation, flattening, and a surface-based coordinate system. Neuroimage 9, 195-207. https://doi.org/10.1006/nimg.1998.0396

Fischl, B., Sereno, M.I., Tootell, R.B., Dale, A.M., 1999b. High-resolution intersubject averaging and a coordinate system for the cortical surface. Hum. Brain Mapp. 8, 272-284.

Fox, M.D., Snyder, A.Z., Vincent, J.L., Corbetta, M., Van Essen, D.C., Raichle, M.E., 2005. The Human Brain Is Intrinsically Organized into Dynamic, Anticorrelated Functional The human brain is intrinsically organized into dynamic, anticorrelated functional networks. Source Proc. Natl. Acad. 
Sci. United States Am. 102, 9673-9678.

Frässle, S., Aponte, E.A., Bollmann, S., Brodersen, K.H., Do, C.T., Harrison, O.K., Harrison, S.J., Heinzle, J., Iglesias, S., Kasper, L., Lomakina, E.I., Mathys, C., Müller-Schrader, M., Pereira, I., Petzschner, F.H., Raman, S., Schöbi, D., Toussaint, B., Weber, L.A., Yao, Y., Stephan, K.E., 2021 a. TAPAS: An Open-Source Software Package for Translational Neuromodeling and Computational Psychiatry. Front. Psychiatry 12. https://doi.org/10.3389/fpsyt.2021.680811

Frässle, S., Harrison, S.J., Heinzle, J., Clementz, B.A., Tamminga, C.A., Sweeney, J.A., Gershon, E.S., Keshavan, M.S., Pearlson, G.D., Powers, A., Stephan, K.E., 2021b. Regression dynamic causal modeling for resting-state fMRI. Hum. Brain Mapp. hbm.25357. https://doi.org/10.1002/hbm.25357

Frässle, S., Lomakina, E.I., Razi, A., Friston, K.J., Buhmann, J.M., Stephan, K.E., 2017. Regression DCM for fMRI. Neuroimage 155, 406-421. https://doi.org/10.1016/j.neuroimage.2017.02.090

Frith, C.D., Frith, U., 2012. Mechanisms of social cognition. Annu. Rev. Psychol. 63, 287-313. https://doi.org/10.1146/annurev-psych-120710-100449

Fukuchi-Shimogori, T., Grove, E.A., 2001. Neocortex patterning by the secreted signaling molecute FGF8. Science (80-. ). 294, 1071-1074. https://doi.org/10.1126/science.1064252

Fulcher, B.D., Murray, J.D., Zerbi, V., Wang, X.-J., 2019. Multimodal gradients across mouse cortex. Proc. Natl. Acad. Sci. 116, 4689-4695. https://doi.org/10.1073/pnas.1814144116

Gadelmawla, E.S., Koura, M.M., Maksoud, T.M.A., Elewa, I.M., Soliman, H.H., 2002a. Roughness parameters. J. Mater. Process. Technol. 123, 133-145. https://doi.org/10.1016/S09240136(02)00060-2

Gadelmawla, E.S., Koura, M.M., Maksoud, T.M.A., Elewa, I.M., Soliman, H.H., 2002b. Roughness parameters. J. Mater. Process. Technol. 123, 133-145. https://doi.org/10.1016/S09240136(02)00060-2

Gao, R., Van den Brink, R.L., Pfeffer, T., Voytek, B., 2020. Neuronal timescales are functionally dynamic and shaped by cortical microarchitecture. Elife 9, 1-44. https://doi.org/10.7554/eLife.61277

García-Cabezas, M.Á., Hacker, J.L., Zikopoulos, B., 2020. A Protocol for Cortical Type Analysis of the Human Neocortex Applied on Histological Samples, the Atlas of Von Economo and Koskinas, and Magnetic Resonance Imaging. Front. Neuroanat. 14, 97. https://doi.org/10.3389/fnana.2020.576015

Glasser, M.F., Coalson, T.S., Bijsterbosch, J.D., Harrison, S.J., Harms, M.P., Anticevic, A., Van Essen, D.C., Smith, S.M., 2018. Using temporal ICA to selectively remove global noise while preserving global signal in functional MRI data. Neuroimage 181, 692-717. https://doi.org/10.1016/j.neuroimage.2018.04.076

Glasser, M.F., Sotiropoulos, S.N., Wilson, J.A., Coalson, T.S., Fischl, B., Andersson, J.L., Xu, J., Jbabdi, S., Webster, M., Polimeni, J.R., Van Essen, D.C., Jenkinson, M., 2013. The minimal preprocessing pipelines for the Human Connectome Project. Neuroimage 80, 105-124.

https://doi.org/10.1016/J.NEUROIMAGE.2013.04.127

Glasser, M.F., Van Essen, D.C., 2011. Mapping human cortical areas in vivo based on myelin content as revealed by T1- and T2-weighted MRI. J. Neurosci. 31, 11597-616. https://doi.org/10.1523/JNEUROSCI.2180-11.2011

Goldman-Rakic, P.S., Schwartz, M.L., 1982. Interdigitation of contralateral and ipsilateral columnar projections to frontal association cortex in primates. Science 216, 755-7. 
Gollo, L.L., Zalesky, A., Hutchison, R.M., Heuvel, M. van den, Breakspear, M., 2015. Dwelling quietly in the rich club: brain network determinants of slow cortical fluctuations. Philos. Trans. R. Soc. B Biol. Sci. 370. https://doi.org/10.1098/RSTB.2014.0165

Goulas, A., Margulies, D.S., Bezgin, G., Hilgetag, C.C., 2019. The architecture of mammalian cortical connectomes in light of the theory of the dual origin of the cerebral cortex. Cortex 118, 244-261. https://doi.org/10.1016/j.cortex.2019.03.002

Goulas, A., Zilles, K., Hilgetag, C.C., 2018. Cortical Gradients and Laminar Projections in Mammals. Trends Neurosci. https://doi.org/10.1016/j.tins.2018.06.003

Greicius, M.D., Supekar, K., Menon, V., Dougherty, R.F., 2009. Resting-state functional connectivity reflects structural connectivity in the default mode network. Cereb. Cortex 19, 72-78. https://doi.org/10.1093/cercor/bhn059

Greve, D.N., Fischl, B., 2009. Accurate and robust brain image alignment using boundary-based registration. Neuroimage 48, 63-72. https://doi.org/10.1016/j.neuroimage.2009.06.060

He, Y., Wang, J., Wang, L., Chen, Z.J., Yan, C., Yang, H., Tang, H., Zhu, C., Gong, Q., Zang, Y., Evans, A.C., 2009. Uncovering Intrinsic Modular Organization of Spontaneous Brain Activity in Humans. PLoS One 4, e5226. https://doi.org/10.1371/journal.pone.0005226

Hilgetag, C.C., Grant, S., 2010. Cytoarchitectural differences are a key determinant of laminar projection origins in the visual cortex. Neuroimage 51, 1006-1017.

https://doi.org/10.1016/j.neuroimage.2010.03.006

Hong, S.-J., Vos de Wael, R., Bethlehem, R.A.I., Lariviere, S., Paquola, C., Valk, S.L., Milham, M.P., Di Martino, A., Margulies, D.S., Smallwood, J., Bernhardt, B.C., 2019. Atypical functional connectome hierarchy in autism. Nat. Commun. 10, 1022. https://doi.org/10.1038/s41467-01908944-1

Horn, A., Ostwald, D., Reisert, M., Blankenburg, F., 2014. The structural-functional connectome and the default mode network of the human brain. Neuroimage 102, 142-151.

https://doi.org/10.1016/j.neuroimage.2013.09.069

Ito, T., Hearne, L.J., Cole, M.W., 2020. A cortical hierarchy of localized and distributed processes revealed via dissociation of task activations, connectivity changes, and intrinsic timescales. Neuroimage 221, 117141. https://doi.org/10.1016/J.NEUROIMAGE.2020.117141

Jenkinson, M., Beckmann, C.F., Behrens, T.E., Woolrich, M.W., Smith, S.M., 2012. Fsl. Neuroimage 62, 782-790. https://doi.org/10.1016/j.neuroimage.2011.09.015

Jeurissen, B., Tournier, J.-D., Dhollander, T., Connelly, A., Sijbers, J., 2014. Multi-tissue constrained spherical deconvolution for improved analysis of multi-shell diffusion MRI data. Neuroimage 103, 411-426. https://doi.org/10.1016/J.NEUROIMAGE.2014.07.061

Khalsa, S., Mayhew, S.D., Chechlacz, M., Bagary, M., Bagshaw, A.P., 2014. The structural and functional connectivity of the posterior cingulate cortex: Comparison between deterministic and probabilistic tractography for the investigation of structure-function relationships. Neuroimage. https://doi.org/10.1016/j.neuroimage.2013.12.022

Kobayashi, Y., Amaral, D.G., 2003. Macaque monkey retrosplenial cortex: II. Cortical afferents. J. Comp. Neurol. 466, 48-79. https://doi.org/10.1002/cne.10883

Kong, R., Li, J., Orban, C., Sabuncu, M.R., Liu, H., Schaefer, A., Sun, N., Zuo, X.N., Holmes, A.J., Eickhoff, S.B., Yeo, B.T.T., 2019. Spatial Topography of Individual-Specific Cortical Networks 
Predicts Human Cognition, Personality, and Emotion. Cereb. Cortex 29, 2533-2551. https://doi.org/10.1093/cercor/bhy123

Leech, R., Scott, G., Carhart-Harris, R., Turkheimer, F., Taylor-Robinson, S.D., Sharp, D.J., 2014. Spatial Dependencies between Large-Scale Brain Networks. PLoS One 9, e98500. https://doi.org/10.1371/journal.pone.0098500

Lehéricy, S., Ducros, M., Moortele, P.-F. Van De, Francois, C., Thivard, L., Poupon, C., Swindale, N., Ugurbil, K., Kim, D.-S., 2004. Diffusion tensor fiber tracking shows distinct corticostriatal circuits in humans. Ann. Neurol. 55, 522-529. https://doi.org/10.1002/ANA.20030

Lepage, C., Mohlberg, H., Pietrzyk, U., Amunts, K., Zilles, K., Evans, A.., 2010. Automatic repair of acquisition defects in reconstruction of histology slices of the human brain, in: 16th Annual Meeting of the Organization for Human Brain Mapping (OHBM). Barcelona.

Lewis, L.B., Lepage, C., Marc, F., Zilles, K., Amunts, K., Evans, A.C., 2014. BigBrain: Initial Tissue Classification and Surface Extraction, in: Annual Meeting of the Organization for Human Brain Mapping. Hamburg.

Lewis, L.B., Lepage, C.Y., Glasser, M.F., Coalson, T.S., Van Essen, D.S., Evans, A.C., 2020. A multimodal surface matching (MSM) surface registration pipeline to bridge atlases across the MNI and the Freesurfer/Human Connectome Project Worlds, in: Annual Meeting of the Organization for Human Brain Mapping.

Li, Q., Tavakol, S., Royer, J., Larivière, S., Vos De Wael, R., Park, B., Paquola, C., Zeng, D., Caldairou, B., Bassett, D.S., Bernasconi, A., Bernasconi, N., Frauscher, B., Smallwood, J., Caciagli, L., Li, S., Bernhardt, B.C., 2021. Atypical neural topographies underpin dysfunctional pattern separation in temporal lobe epilepsy. Brain. https://doi.org/10.1093/brain/awab121

Mar, R.A., 2011. The Neural Bases of Social Cognition and Story Comprehension. Annu. Rev. Psychol. 62, 103-134. https://doi.org/10.1146/annurev-psych-120709-145406

Margulies, D.S., Ghosh, S.S., Goulas, A., Falkiewicz, M., Huntenburg, J.M., Langs, G., Bezgin, G., Eickhoff, S.B., Castellanos, F.X., Petrides, M., Jefferies, E., Smallwood, J., 2016. Situating the default-mode network along a principal gradient of macroscale cortical organization. Proc. Natl. Acad. Sci. 113, 12574-12579. https://doi.org/10.1073/pnas.1608282113

Margulies, D.S., Vincent, J.L., Kelly, C., Lohmann, G., Uddin, L.Q., Biswal, B.B., Villringer, A., Castellanos, F.X., Milham, M.P., Petrides, M., 2009. Precuneus shares intrinsic functional architecture in humans and monkeys. Proc. Natl. Acad. Sci. U. S. A. 106, 20069-74. https://doi.org/10.1073/pnas.0905314106

McCormick, C., Protzner, A.B., Barnett, A.J., Cohn, M., Valiante, T.A., McAndrews, M.P., 2014. Linking DMN connectivity to episodic memory capacity: What can we learn from patients with medial temporal lobe damage? NeuroImage Clin. 5, 188-196.

https://doi.org/10.1016/j.nicl.2014.05.008

McFarland, N.R., Haber, S.N., 2000. Convergent Inputs from Thalamic Motor Nuclei and Frontal Cortical Areas to the Dorsal Striatum in the Primate. J. Neurosci. 20, 3798. https://doi.org/10.1523/JNEUROSCI.20-10-03798.2000

McGarigal, K., Tagil, S., Cushman, S.A., 2009. Surface metrics: an alternative to patch metrics for the quantification of landscape structure. Landsc. Ecol. 2009243 24, 433-450. https://doi.org/10.1007/S10980-009-9327-Y

Merker, B., 1983. Silver staining of cell bodies by means of physical development. J. Neurosci. Methods 


\section{9, 235-241. https://doi.org/10.1016/0165-0270(83)90086-9}

Mesulam, M.-M., 1998. From sensation to cognition. Brain 121, 1013-1052. https://doi.org/10.1093/brain/121.6.1013

Mesulam, M. -M, 1990. Large-scale neurocognitive networks and distributed processing for attention, language, and memory. Ann. Neurol. 28, 597-613. https://doi.org/10.1002/ana.410280502

Mohlberg, H., Tweddell, B., Lippert, T., Amunts, K., 2016. Workflows for Ultra-High Resolution 3D Models of the Human Brain on Massively Parallel Supercomputers. Springer, Cham, pp. 15-27. https://doi.org/10.1007/978-3-319-50862-7_2

Molnar-Szakacs, I., Uddin, L.Q., 2013. Self-processing and the default mode network: Interactions with the mirror neuron system. Front. Hum. Neurosci. https://doi.org/10.3389/fnhum.2013.00571

Murphy, C., Jefferies, E., Rueschemeyer, S.A., Sormaz, M., Wang, H. ting, Margulies, D.S., Smallwood, J., 2018. Distant from input: Evidence of regions within the default mode network supporting perceptually-decoupled and conceptually-guided cognition. Neuroimage 171, 393-401. https://doi.org/10.1016/j.neuroimage.2018.01.017

Murphy, C., Wang, H.T., Konu, D., Lowndes, R., Margulies, D.S., Jefferies, E., Smallwood, J., 2019. Modes of operation: A topographic neural gradient supporting stimulus dependent and independent cognition. Neuroimage 186, 487-496. https://doi.org/10.1016/j.neuroimage.2018.11.009

Murray, J.D., Bernacchia, A., Freedman, D.J., Romo, R., Wallis, J.D., Cai, X., Padoa-Schioppa, C., Pasternak, T., Seo, H., Lee, D., Wang, X.-J., 2014. A hierarchy of intrinsic timescales across primate cortex. Nat. Neurosci. 17, 1661-3. https://doi.org/10.1038/nn.3862

Muscoloni, A., Thomas, J.M., Ciucci, S., Bianconi, G., Cannistraci, C.V., 2017. Machine learning meets complex networks via coalescent embedding in the hyperbolic space. Nat. Commun. 8, 1-19. https://doi.org/10.1038/s41467-017-01825-5

Ossandón, T., Jerbi, K., Vidal, J.R., Bayle, D.J., Henaff, M.A., Jung, J., Minotti, L., Bertrand, O., Kahane, P., Lachaux, J.P., 2011. Transient suppression of broadband gamma power in the default-mode network is correlated with task complexity and subject performance. J. Neurosci. 31, 14521-14530. https://doi.org/10.1523/JNEUROSCI.2483-11.2011

Pandya, D., Seltzer, B., Petrides, M., Cipolloni, P.B., 2015. Cerebral Cortex: Architecture, Connections and the Dual Origin Concept. Oxford University Press. https://doi.org/10.1093/med/9780195385151.001.0001

Pandya, D.N., Yeterian, E.H., 1985. Architecture and Connections of Cortical Association Areas. Springer, Boston, MA, pp. 3-61. https://doi.org/10.1007/978-1-4757-9619-3_1

Paquola, C., Benkarim, O., Dekraker, J., Larivière, S., Frässle, S., Royer, J., Tavakol, S., Valk, S., Bernasconi, A., Bernasconi, N., Khan, A., Evans, A.C., Razi, A., Smallwood, J., Bernhardt, B.C., 2020. Convergence of cortical types and functional motifs in the human mesiotemporal lobe. Elife 9 , 1-26. https://doi.org/10.7554/eLife.60673

Paquola, C., Bethlehem, R.A., Seidlitz, J., Wagstyl, K., Romero-Garcia, R., Whitaker, K.J., Vos de Wael, R., Williams, G.B., Vértes, P.E., Margulies, D.S., Bernhardt, B., Bullmore, E.T., 2019a. Shifts in myeloarchitecture characterise adolescent development of cortical gradients. Elife 8. https://doi.org/10.7554/eLife.50482

Paquola, C., Royer, J., Lewis, L.B., Lepage, C., Glatard, T., Wagstyl, K., DeKraker, J., Toussaint, P.-J., Valk, S.L., Collins, D.L., Khan, A., Amunts, K., Evans, A.C., Dickscheid, T., Bernhardt, B.C., 
2021. The BigBrainWarp toolbox for integration of BigBrain 3D histology with multimodal neuroimaging. Elife 10. https://doi.org/10.7554/ELIFE.70119

Paquola, C., Vos De Wael, R., Wagstyl, K., Bethlehem, R.A.I., Hong, S.-J., Seidlitz, J., Bullmore, E.T., Evans, A.C., Misic, B., Margulies, D.S., Smallwood, J., Bernhardt, B.C., 2019b. Microstructural and functional gradients are increasingly dissociated in transmodal cortices. PLoS Biol. 17, e3000284. https://doi.org/10.1371/journal.pbio.3000284

Pedregosa, F., Varoquaux, G., Gramfort, A., Thirion, B., Grisel, O., Dubourg, V., Passos, A., Brucher, M., Perrot, M., Duchesnay, andÉdouard, 2011. Scikit-learn: Machine Learning in Python Gaël Varoquaux Bertrand Thirion Vincent Dubourg Alexandre Passos PEDREGOSA, VAROQUAUX, GRAMFORT ET AL. Matthieu Perrot, Journal of Machine Learning Research.

Raichle, M.E., 2015. The brain's default mode network. Annu Rev Neurosci 38, 433-447. https://doi.org/10.1146/annurev-neuro-071013-014030

Raichle, M.E., MacLeod, A.M., Snyder, A.Z., Powers, W.J., Gusnard, D.A., Shulman, G.L., 2001. A default mode of brain function. Proc. Natl. Acad. Sci. U. S. A. 98, 676-682. https://doi.org/10.1073/pnas.98.2.676

Robinson, E.C., Garcia, K., Glasser, M.F., Chen, Z., Coalson, T.S., Makropoulos, A., Bozek, J., Wright, R., Schuh, A., Webster, M., Hutter, J., Price, A., Cordero Grande, L., Hughes, E., Tusor, N., Bayly, P. V., Van Essen, D.C., Smith, S.M., Edwards, A.D., Hajnal, J., Jenkinson, M., Glocker, B., Rueckert, D., 2018. Multimodal surface matching with higher-order smoothness constraints. Neuroimage 167, 453-465. https://doi.org/10.1016/j.neuroimage.2017.10.037

Robinson, E.C., Jbabdi, S., Glasser, M.F., Andersson, J., Burgess, G.C., Harms, M.P., Smith, S.M., Van Essen, D.C., Jenkinson, M., 2014. MSM: A new flexible framework for multimodal surface matching. Neuroimage 100, 414-426. https://doi.org/10.1016/j.neuroimage.2014.05.069

Royer, J., Rodríguez-Cruces, R., Tavakol, S., Larivière, S., Herholz, P., Li, Q., Wael, R.V. de, Paquola, C., Benkarim, O., Park, B., Lowe, A.J., Margulies, D., Smallwood, J., Bernasconi, A., Bernasconi, N., Frauscher, B., Bernhardt, B.C., 2021. An Open MRI Dataset for Multiscale Neuroscience. bioRxiv 2021.08.04.454795. https://doi.org/10.1101/2021.08.04.454795

Rumelhart, D.E., McClelland, J.L., 1987. The Appeal of Parallel Distributed Processing, in: Parallel Distributed Processing: Explorations in the Microstructure of Cognition: Foundations. MIT Presss, pp. 3-44.

Rumelhart, D.E., McClelland, J.L., 1986. Parallel distributed processing : explorations in the microstructure of cognition. MIT Press, Cambridge, Mass.

Salimi-Khorshidi, G., Douaud, G., Beckmann, C.F., Glasser, M.F., Griffanti, L., Smith, S.M., 2014. Automatic denoising of functional MRI data: Combining independent component analysis and hierarchical fusion of classifiers. Neuroimage 90, 449-468. https://doi.org/10.1016/j.neuroimage.2013.11.046

Sandhu, Z., Tanglay, O., Young, I.M., Briggs, R.G., Bai, M.Y., Larsen, M.L., Conner, A.K., Dhanaraj, V., Lin, Y.H., Hormovas, J., Fonseka, R.D., Glenn, C.A., Sughrue, M.E., 2020. Parcellation-based anatomic modeling of the default mode network. Brain Behav. 11, e01976. https://doi.org/10.1002/brb3.1976

Schaefer, A., Kong, R., Gordon, E.M., Laumann, T.O., Zuo, X.-N., Holmes, A.J., Eickhoff, S.B., Yeo, B.T.T., 2018. Local-Global Parcellation of the Human Cerebral Cortex from Intrinsic Functional Connectivity MRI. Cereb. Cortex 28, 3095-3114. https://doi.org/10.1093/cercor/bhx179 
Schleicher, A., Amunts, K., Geyer, S., Morosan, P., Zilles, K., 1999. Observer-Independent Method for Microstructural Parcellation of Cerebral Cortex: A Quantitative Approach to Cytoarchitectonics. Neuroimage 9, 165-177. https://doi.org/10.1006/nimg.1998.0385

Scholtens, L.H., de Reus, M.A., de Lange, S.C., Schmidt, R., van den Heuvel, M.P., 2016. An MRI Von Economo - Koskinas atlas. Neuroimage. https://doi.org/10.1016/j.neuroimage.2016.12.069

Seguin, C., Razi, A., Zalesky, A., 2019. Inferring neural signalling directionality from undirected structural connectomes. Nat. Commun. 10, 1-13. https://doi.org/10.1038/s41467-019-12201-w

Seguin, C., Van Den Heuvel, M.P., Zalesky, A., 2018. Navigation of brain networks. Proc. Natl. Acad. Sci. U. S. A. 115, 6297-6302. https://doi.org/10.1073/pnas.1801351115

Seitzman, B.A., Gratton, C., Laumann, T.O., Gordon, E.M., Adeyemo, B., Dworetsky, A., Kraus, B.T., Gilmore, A.W., Berg, J.J., Ortega, M., Nguyen, A., Greene, D.J., McDermott, K.B., Nelson, S.M., Lessov-Schlaggar, C.N., Schlaggar, B.L., Dosenbach, N.U.F., Petersen, S.E., 2019. Trait-like variants in human functional brain networks. Proc. Natl. Acad. Sci. U. S. A. 116, 22851-22861. https://doi.org/10.1073/pnas.1902932116

Selemon, L.D., Goldman-Rakic, P.S., 1988. Common Cortical and Subcortical Targets of the Dorsolateral Prefrontal and Posterior Parietal Cortices in the Rhesus Monkey: Evidence for a Distributed Neural Network Subserving Spatially Guided Behavior, The Journal of Neuroscience.

Selemon, L.D., Goldman-Rakic, P.S., 1985. Longitudinal topography and interdigitation of corticostriatal projections in the rhesus monkey. J. Neurosci. 5, 776-794. https://doi.org/10.1523/jneurosci.05-0300776.1985

Sepulcre, J., Sabuncu, M.R., Yeo, T.B., Liu, H., Johnson, K.A., 2012. Stepwise connectivity of the modal cortex reveals the multimodal organization of the human brain. J. Neurosci. 32, 10649-10661. https://doi.org/10.1523/JNEUROSCI.0759-12.2012

Sestieri, C., Corbetta, M., Romani, G.L., Shulman, G.L., 2011. Episodic memory retrieval, parietal cortex, and the default mode network: Functional and topographic analyses. J. Neurosci. 31, 44074420. https://doi.org/10.1523/JNEUROSCI.3335-10.2011

Shine, J.M., 2021. The thalamus integrates the macrosystems of the brain to facilitate complex, adaptive brain network dynamics. Prog. Neurobiol. 199, 101951. https://doi.org/10.1016/j.pneurobio.2020.101951

Shulman, G.L., Fiez, J.A., Corbetta, M., Buckner, R.L., Miezin, F.M., Raichle, M.E., Petersen, S.E., 1997. Common Blood Flow Changes across Visual Tasks: II. Decreases in Cerebral Cortex. J. Cogn. Neurosci. 9, 648-663. https://doi.org/10.1162/jocn.1997.9.5.648

Simony, E., Honey, C.J., Chen, J., Lositsky, O., Yeshurun, Y., Wiesel, A., Hasson, U., 2016. Dynamic reconfiguration of the default mode network during narrative comprehension. Nat. Commun. 7 , 12141. https://doi.org/10.1038/ncomms 12141

Smallwood, J., Bernhardt, B.C., Leech, R., Bzdok, D., Jefferies, E., Margulies, D.S., 2021a. The default mode network in cognition: a topographical perspective. Nat. Rev. Neurosci. 2021 1-11. https://doi.org/10.1038/s41583-021-00474-4

Smallwood, J., Bernhardt, B.C., Leech, R., Bzdok, D., Jefferies, E., Margulies, D.S., 2021b. The default mode network in cognition: a topographical perspective. Nat. Rev. Neurosci. 2021228 22, 503-513. https://doi.org/10.1038/s41583-021-00474-4

Smith, R.E., Tournier, J.-D., Calamante, F., Connelly, A., 2012. Anatomically-constrained tractography: 
improved diffusion MRI streamlines tractography through effective use of anatomical information. Neuroimage 62, 1924-38. https://doi.org/10.1016/j.neuroimage.2012.06.005

Smith, R.E., Tournier, J.D., Calamante, F., Connelly, A., 2015. SIFT2: Enabling dense quantitative assessment of brain white matter connectivity using streamlines tractography. Neuroimage 119, 338-351. https://doi.org/10.1016/j.neuroimage.2015.06.092

Spreng, R.N., Stevens, W.D., Chamberlain, J.P., Gilmore, A.W., Schacter, D.L., 2010. Default network activity, coupled with the frontoparietal control network, supports goal-directed cognition. Neuroimage 53, 303-317. https://doi.org/10.1016/j.neuroimage.2010.06.016

Strobl, C., Boulesteix, A.L., Kneib, T., Augustin, T., Zeileis, A., 2008. Conditional variable importance for random forests. BMC Bioinformatics 9, 1-11. https://doi.org/10.1186/1471-2105-9-307

Taubin, G., 1995. Curve and surface smoothing without shrinkage, in: IEEE International Conference on Computer Vision. IEEE, pp. 852-857. https://doi.org/10.1109/iccv.1995.466848

Tenenbaum, J.B., De Silva, V., Langford, J.C., 2000. A global geometric framework for nonlinear dimensionality reduction. Science (80-. ). 290, 2319-23.

https://doi.org/10.1126/science.290.5500.2319

The MathWorks, I., 2019. MATLAB and Statistics Toolbox Release.

Tournier, J.-D., Calamante, F., Connelly, A., 2012. MRtrix: diffusion tractography in crossing fiber regions. Int. J. Imaging Syst. Technol. 22, 53-66.

Tournier, J.-D., Smith, R., Raffelt, D., Tabbara, R., Dhollander, T., Pietsch, M., Christiaens, D., Jeurissen, B., Yeh, C.-H., Connelly, A., 2019. MRtrix3: A fast, flexible and open software framework for medical image processing and visualisation. Neuroimage 116137.

Tournier, J.D., Calamante, F., Connelly, A., 2007. Robust determination of the fibre orientation distribution in diffusion MRI: Non-negativity constrained super-resolved spherical deconvolution. Neuroimage 35, 1459-1472. https://doi.org/10.1016/j.neuroimage.2007.02.016

Tustison, N.J., Avants, B.B., 2013. Explicit B-spline regularization in diffeomorphic image registration. Front. Neuroinform. 7, 39. https://doi.org/10.3389/fninf.2013.00039

Tustison, N.J., Avants, B.B., Cook, P.A., Zheng, Y., Egan, A., Yushkevich, P.A., Gee, J.C., 2010. N4ITK: Improved N3 Bias Correction. IEEE Trans. Med. Imaging 29, 1310-1320. https://doi.org/10.1109/TMI.2010.2046908

Uddin, L.Q., Kelly, A.M.C., Biswal, B.B., Castellanos, F.X., Milham, M.P., 2009. Functional Connectivity of Default Mode Network Components: Correlation, Anticorrelation, and Causality. Hum. Brain Mapp. 30, 625-637. https://doi.org/10.1002/hbm.20531

Van Den Heuvel, M., Mandl, R., Luigjes, J., Pol, H.H., 2008. Microstructural organization of the cingulum tract and the level of default mode functional connectivity. J. Neurosci. 28, 10844-10851. https://doi.org/10.1523/JNEUROSCI.2964-08.2008

van den Heuvel, M.P., Sporns, O., 2011. Rich-Club Organization of the Human Connectome. J. Neurosci. 31, 15775-15786. https://doi.org/10.1523/JNEUROSCI.3539-11.2011

Van Essen, D.C., Smith, S.M., Barch, D.M., Behrens, T.E.J., Yacoub, E., Ugurbil, K., 2013. The WUMinn Human Connectome Project: An overview. Neuroimage 80, 62-79. https://doi.org/10.1016/j.neuroimage.2013.05.041

Van Oort, E.S.B., van Cappellen van Walsum, A.M., Norris, D.G., 2014. An investigation into the 
functional and structural connectivity of the Default Mode Network. Neuroimage 90, 381-389. https://doi.org/10.1016/j.neuroimage.2013.12.051

Von Economo, C., Koskinas, G., 1925. Die Cytoarchitektonik der Hirnrinde des erwachsenen Menschen. Springer, Berlin.

Von Economo, C., Triarhou, L.C., 2009. Cellular structure of the human cerebral cortex, Cellular Structure of the Human Cerebral Cortex. S. Karger AG. https://doi.org/10.1093/brain/awp268

Von Luxburg, U., 2007. A tutorial on spectral clustering. Stat. Comput. 17, 395-416. https://doi.org/10.1007/s11222-007-9033-Z

Vos de Wael, R., Benkarim, O., Paquola, C., Lariviere, S., Royer, J., Tavakol, S., Xu, T., Hong, S.-J., Langs, G., Valk, S., Misic, B., Milham, M., Margulies, D., Smallwood, J., Bernhardt, B.C., 2020. BrainSpace: a toolbox for the analysis of macroscale gradients in neuroimaging and connectomics datasets. Commun. Biol. 3, 103. https://doi.org/10.1038/s42003-020-0794-7

Vos de Wael, R., Larivière, S., Caldairou, B., Hong, S.-J., Margulies, D.S., Jefferies, E., Bernasconi, A., Smallwood, J., Bernasconi, N., Bernhardt, B.C., 2018. Anatomical and microstructural determinants of hippocampal subfield functional connectome embedding. Proc. Natl. Acad. Sci. U. S. A. 115, 10154-10159. https://doi.org/10.1073/pnas.1803667115

Waehnert, M.D., Dinse, J., Weiss, M., Streicher, M.N., Waehnert, P., Geyer, S., Turner, R., Bazin, P.-L., 2014. Anatomically motivated modeling of cortical laminae. Neuroimage 93, 210-220. https://doi.org/10.1016/J.NEUROIMAGE.2013.03.078

Wagstyl, K., Lepage, C., Bludau, S., Zilles, K., Fletcher, P.C., Amunts, K., Evans, A.C., 2018a. Mapping Cortical Laminar Structure in the 3D BigBrain. Cereb. Cortex 28, 2551-2562. https://doi.org/10.1093/cercor/bhy074

Wagstyl, K., Paquola, C., Bethlehem, R., Huth, A., 2018b. kwagstyl/surface_tools: Initial release of equivolumetric surfaces. https://doi.org/10.5281/ZENODO.1412054

Worsley, K., Taylor, J., Carbonell, F., Chung, M., Duerden, E., Bernhardt, B., Lyttelton, O., Boucher, M., Evans, A., 2009. SurfStat: A Matlab toolbox for the statistical analysis of univariate andmultivariate surface and volumetric data using linear mixed effects modelsand random field theory, in: Human Brain Mapping.

Yeo, B.T.T., Krienen, F.M., Sepulcre, J., Lashkari, D., Hollinshead, M., Roffman, J.L., Smoller, J.W., Zöllei, L., Polimeni, J.R., Fischl, B., Liu, H., Buckner, R.L., 2011. The organization of the human cerebral cortex estimated by intrinsic functional connectivity. J. Neurophysiol. 106, 1125-1165. https://doi.org/10.1152/jn.00338.2011

Yeshurun, Y., Nguyen, M., Hasson, U., 2021. The default mode network: where the idiosyncratic self meets the shared social world. Nat. Rev. Neurosci. 22, 181-192. https://doi.org/10.1038/s41583020-00420-w

Zilles, K., Schleicher, A., Palomero-Gallagher, N., Amunts, K., 2002. Quantitative Analysis of Cyto- and Receptor Architecture of the Human Brain, in: Brain Mapping: The Methods. Academic Press, pp. 573-602. https://doi.org/10.1016/b978-012693019-1/50023-x 


\section{Supplementary Material}

A | First five eigenvectors of cytoarchitectural differentiation

E1
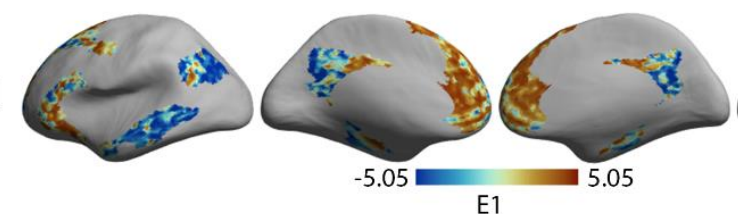

E2
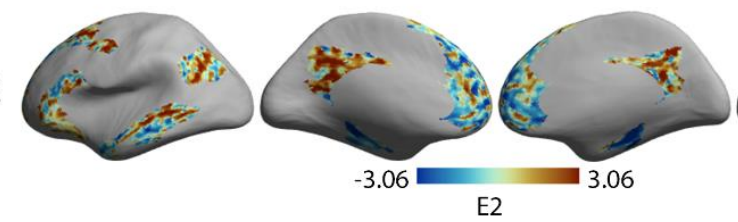

E3
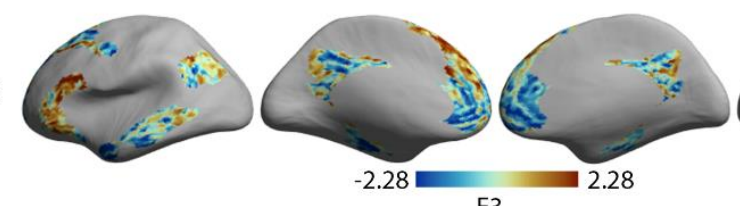

E3

E4
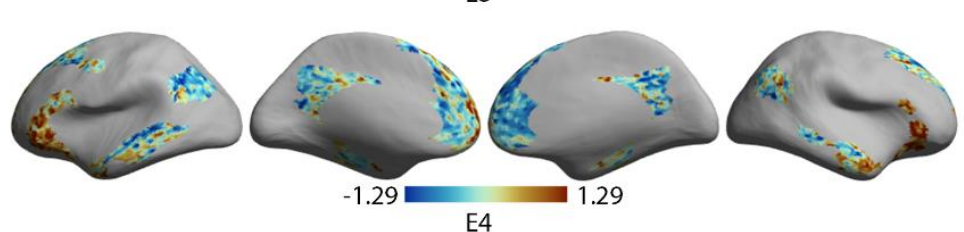

E5
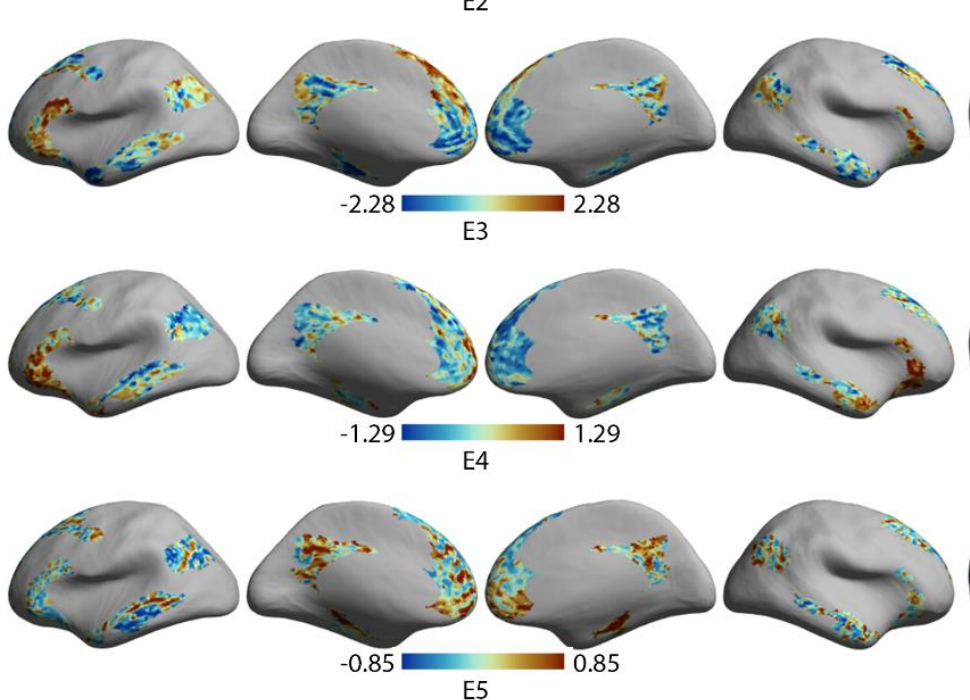

$\mathrm{B}$ | Comparison of data-driven cytoarchitectural axis with cortical types

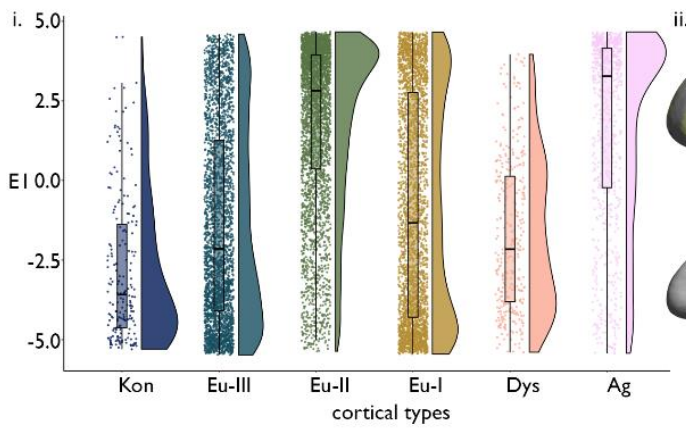

ii.

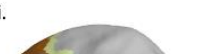

2 6

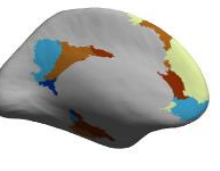

$-5.28$
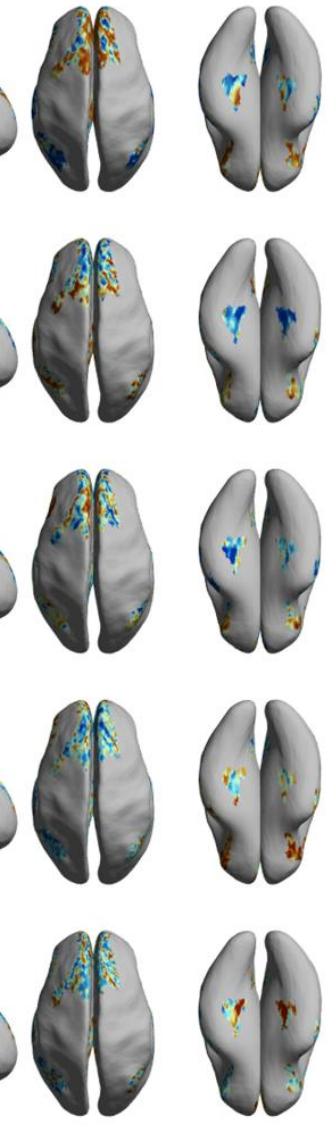

iii.
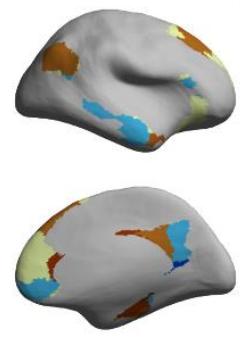

rescaled type
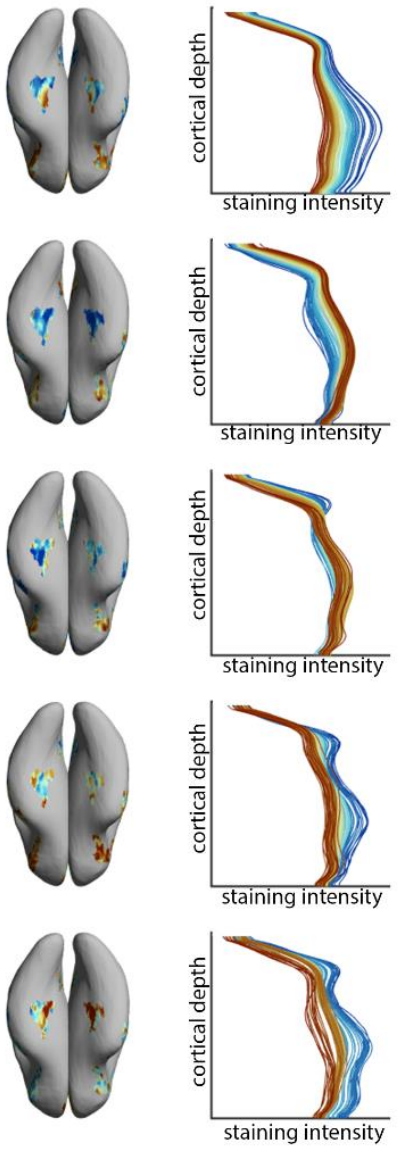

Supplementary Figure 1: A) First five eigenvectors projected on the inflated BigBrain surface. For line plots on the right, staining intensity profiles averaged within 100 bins of the respective eigenvector and coloured by eigenvector position. B) i. E1 projected onto inflated fsaverage surface. ii. Raincloud plot shows the distribution of E1 across cortical types. iii. Cortical type assignment (1:6) was rescaled to the range of E1 (-0.71:0.71) then subtracted from E1, producing a deviation map that highlights where the type-based and data-driven depictions of DMN cytoarchitecture differ. Negative values indicate lower E1 than expected by a linear relationship with cortical type, whereas positive values indicate higher E1. 

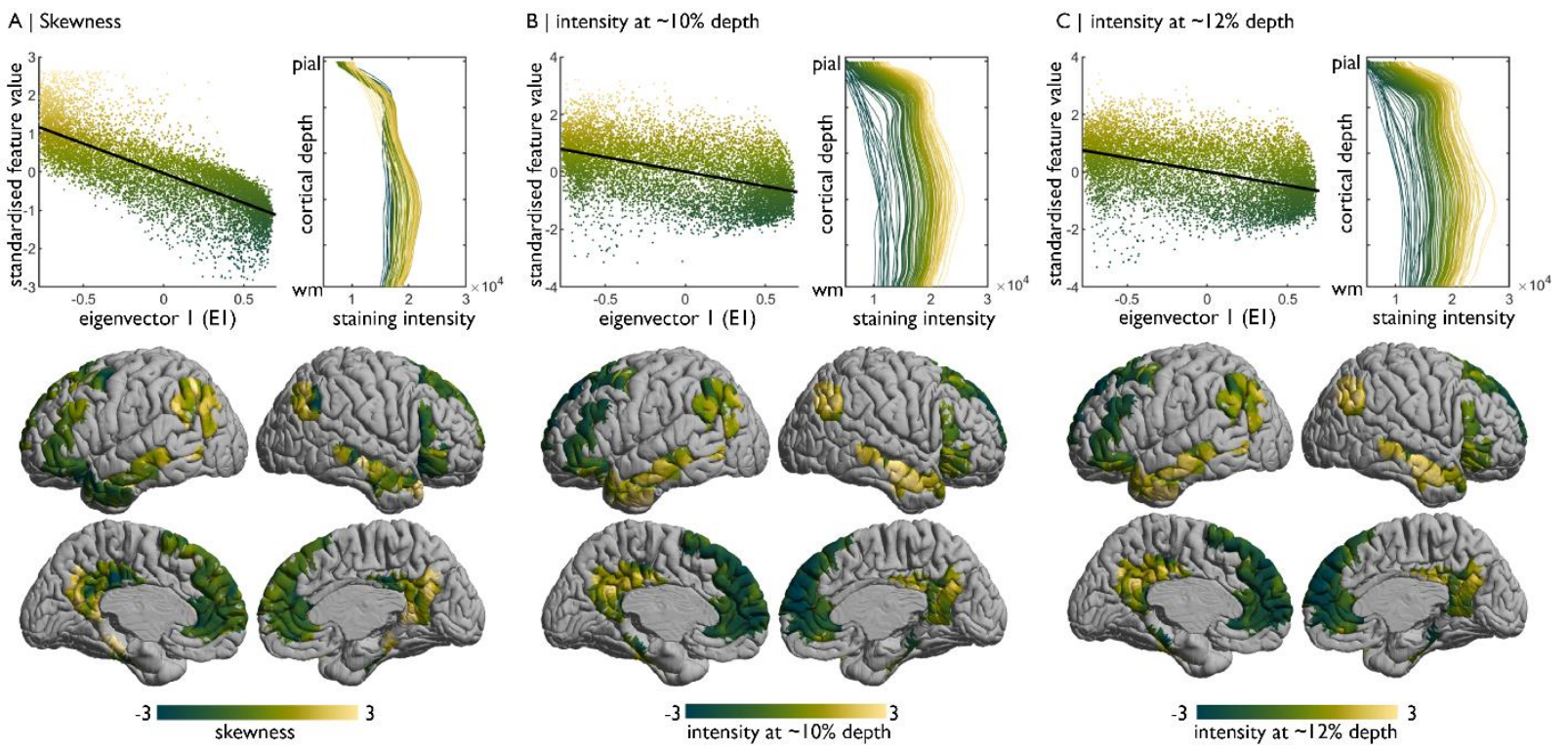

Supplementary Figure 2: Selected staining intensity profile features. Top: Scatterplot shows correlation of standardised feature values with position on eigenvector 1 . Line plots show average staining intensity profiles for 100 bins, stratified by the respective feature value, which illustrate how features capture differences in the profile shape. Profiles are coloured by standardised feature value. Higher Merker-staining intensity values reflect higher cellular density/soma size. Lower: Feature values projected onto the cortical surface.
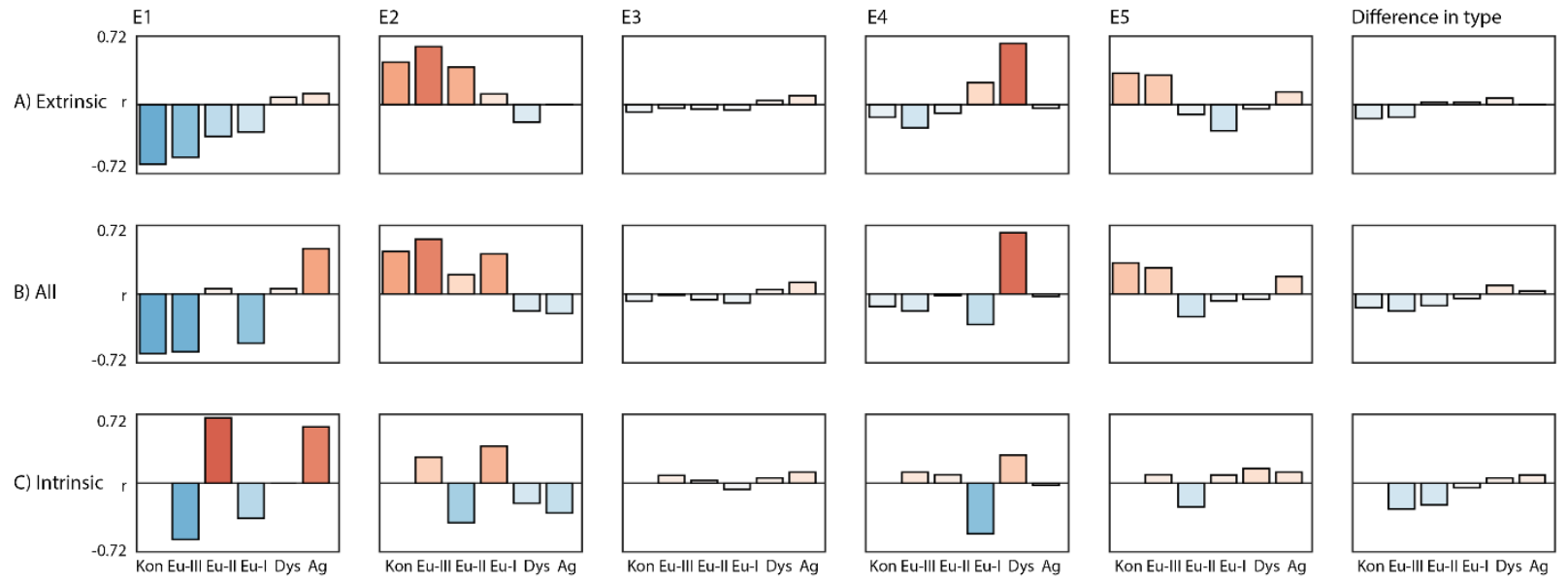

Kon Eu-III Eu-II Eu-I Dys Ag
Kon Eu-III Eu-II Eu-I Dys Ag
Supplementary Figure 3: Influence of cytoarchitecture on navigation efficiency. Bar plots show the linear correlation coefficient (r) of type-average Enav with a cytoarchitectural feature. Each column represents a different cytoarchitectural feature of the DMN. E1-5 are the first five eigenvectors (Supplementary Figure 1). The final column, "Difference in type", is the difference between the DMN seed and target. Type-average Enav is calculated as the average Enav to A) non-DMN, B) all, or C) DMN parcels of a certain type. 
bioRxiv preprint doi: https://doi.org/10.1101/2021.11.22.469533; this version posted November 22, 2021. The copyright holder for this preprint (which was not certified by peer review) is the author/funder, who has granted bioRxiv a license to display the preprint in perpetuity. It is made available under aCC-BY-NC-ND 4.0 International license.
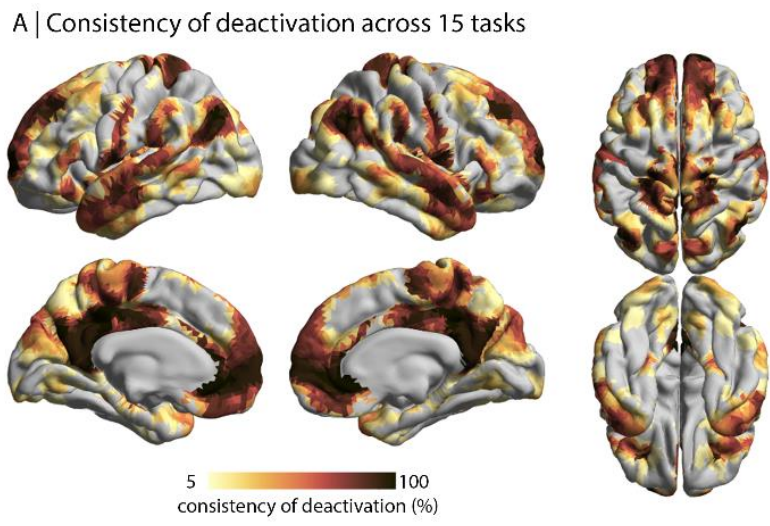

C | Consistency of DMN assignment across 1026 individuals

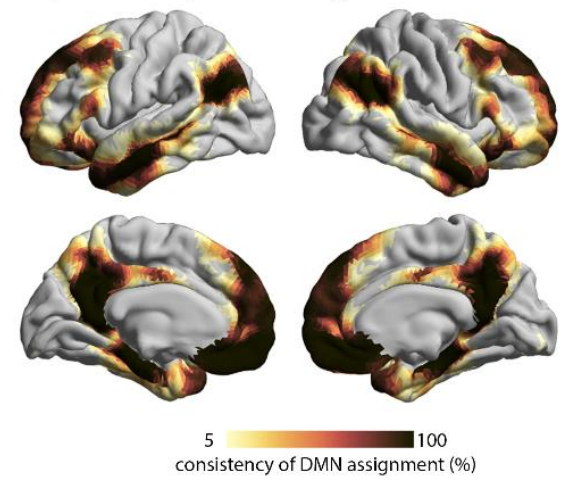

B | Type proportions across consistency thresholds

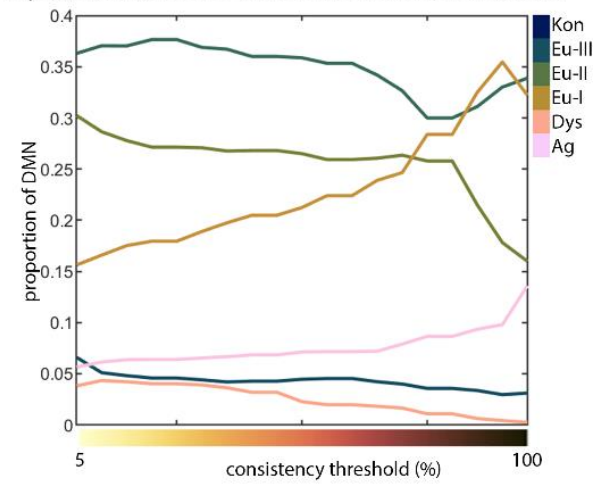

D|Type proportions across consistency thresholds

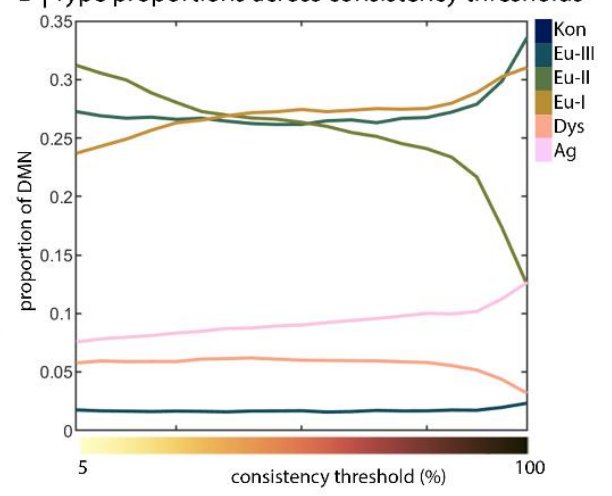

Supplementary Figure 4: Cytoarchitectural heterogeneity of the DMN with alternative atlases. A) DMN based on consistency of deactivation during externally-oriented tasks. Vertex-wise change in the BOLD response were calculated across 787 subjects in Human Connectome Project during fifteen externally-oriented tasks. Surface projections show the consistency of deactivations $(\mathrm{z} \leq-5)$ across the tasks. B) Proportion of the DMN assigned to each cortical type, where the DMN is defined variably based on different consistency thresholds of the map in (A). C) Probability of the DMN at each vertex, calculated across 1029 individualspecific functional network delineations (Kong et al., 2019). B) Proportion of the DMN assigned to each cortical type, where the DMN is defined variably based on different consistency thresholds of the map in (C).
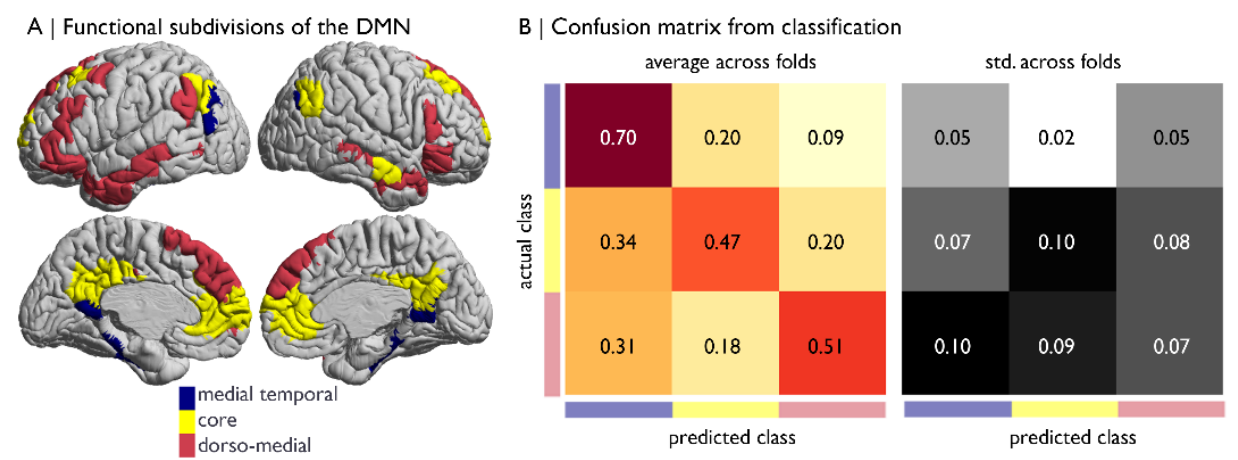

C| Feature importance

Supplementary Figure 5: A) Three subdivisions of the DMN (Yeo et al., 2011). B) Outcome of linear vector classification across four folds. Subdivision membership was predicted by the first ten eigenvectors that resulted from diffusion map embedding on microstructure profile covariance within the DMN. C) Full model predictive accuracy was compared shuffle-onefeature models. The scatter plot shows decrease in accuracy with each feature for each subdivision. Vertical bars indicate standard deviation in decrease in accuracy across folds. 

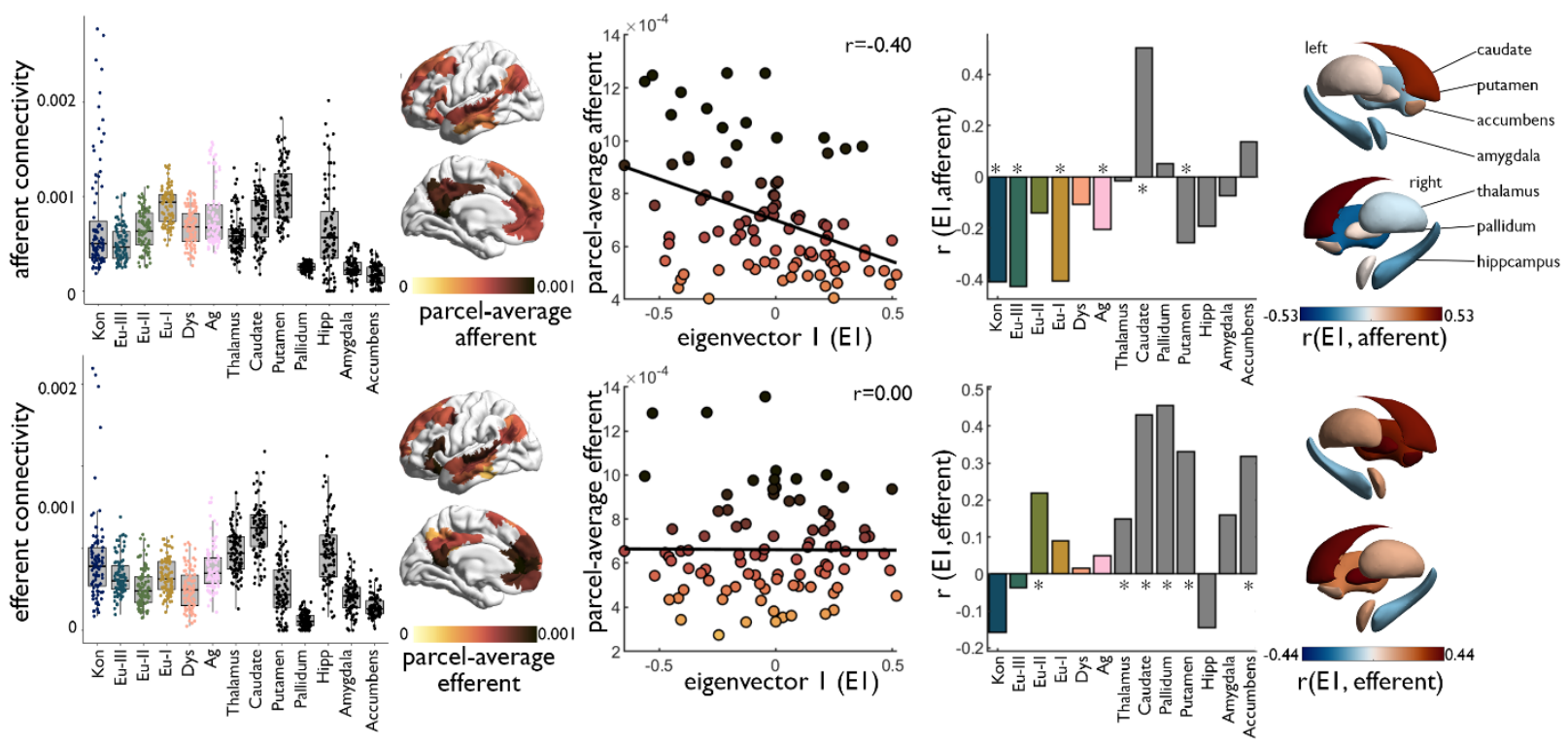

Supplementary Figure 6: Expanded rDCM with non-isocortical regions. Left Boxplots show type-average effective connectivity with the DMN, where each point reflecting a DMN parcel. Centre left Surface plots show the average effective connectivity of each DMN parcel, taken across all non-DMN isocortical parcels. This represents the y-axis in the correlation with parcel-wise values of eigenvector 1 (E1, ie: data-driven cytoarchitectural axis) in the scatterplot. Centre right Bar plots show the correlation coefficient type- or region-average effective connectivity with the cytoarchitectural axis. Asterisks indicate $\mathrm{p}_{\text {spin }}<0.002$ (ie: Bonferroni correction of $\alpha=0.05$ for thirteen two-sided tests). Far right Surface plots show the correlation coefficient of effective connectivity with the cytoarchitectural axis for non-isocortical regions.

i. Cortical type of target

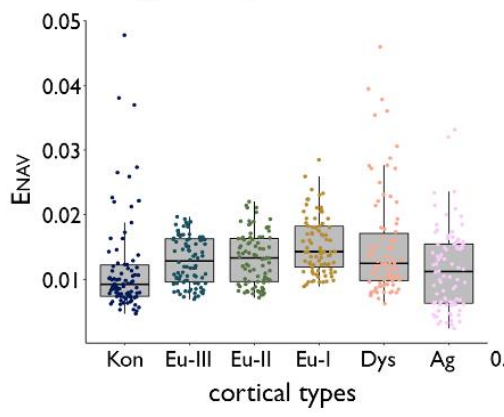

ii. Gradient position of DMN seed

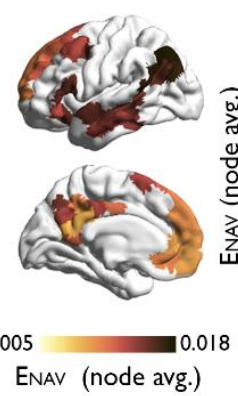

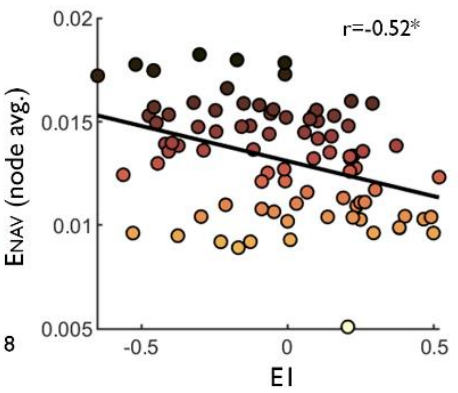

iii. Type by gradient interaction

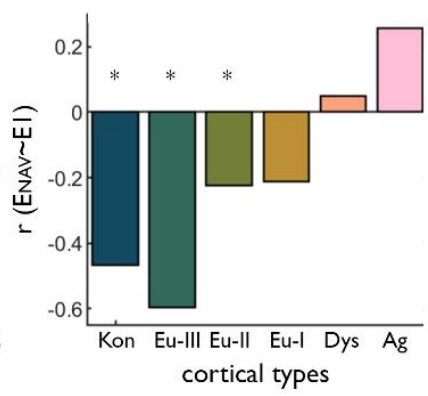

Supplementary Figure 7: DMN structural connectivity organised with respect to cytoarchitecture in the Replication dataset. i. Type-effect: Boxplots show depict average Enav of each DMN parcel to each cortical type. ii. Axis-effect: Cortical surfaces show the average Enav of each DMN parcel. Scatterplot shows the correlation of E1 with Enav. iii. Interaction-effect: Bar plots shows the linear correlation coefficient (r) of E1 value with type-average Enav. 

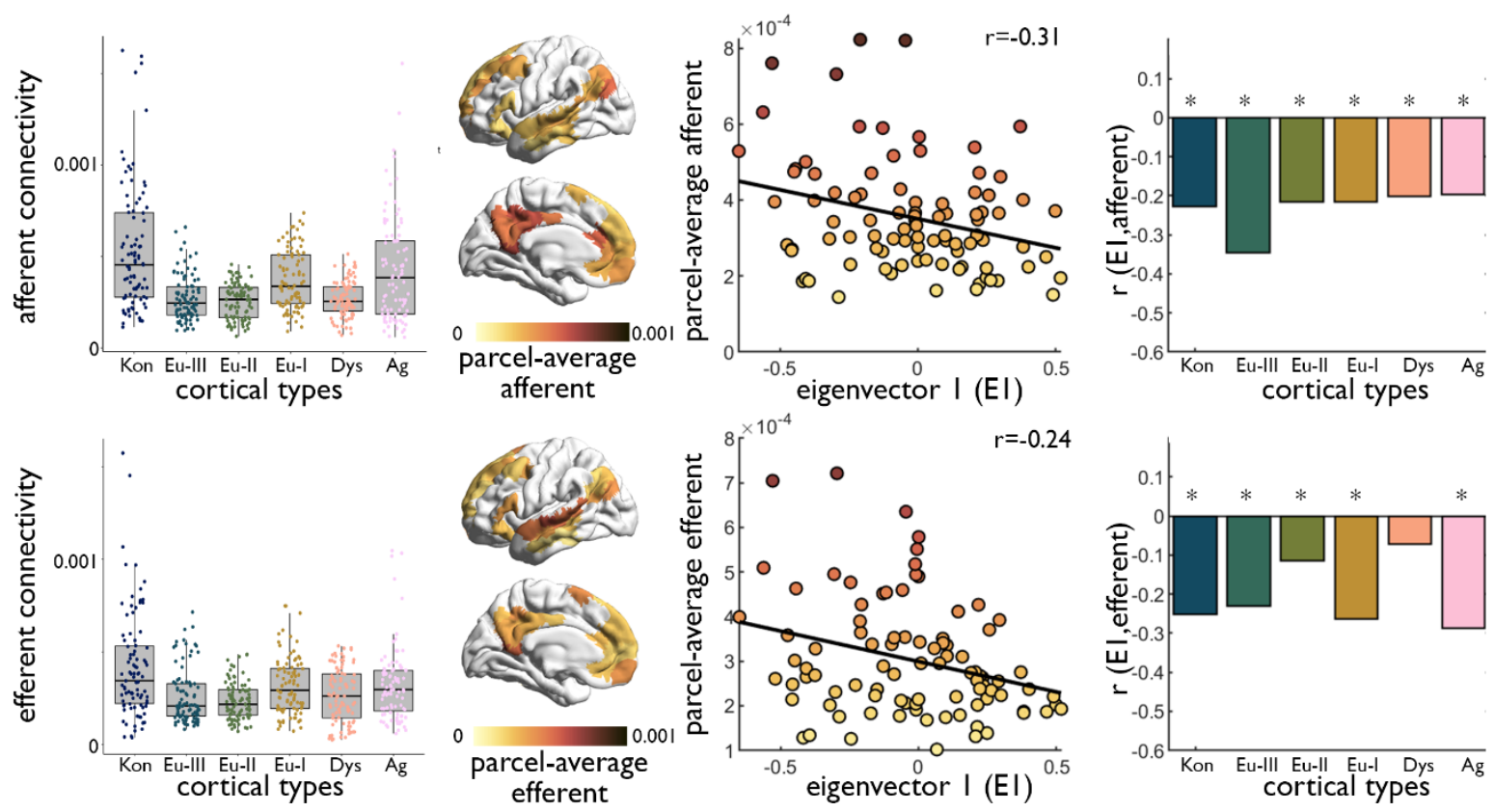

Supplementary Figure 8: Relation of cytoarchitecture to effective connectivity $(E C)$ of the default mode network $(D M N)$ in Replication dataset. Left Boxplots show type-average effective connectivity with the DMN, where each point reflecting a DMN parcel. Centre Surface plots show the average effective connectivity of each DMN parcel, taken across all non-DMN parcels. This represents the $\mathrm{y}$-axis in the correlation with parcel-wise values of the DMN cytoarchitectural axis in the scatterplot. Right Bar plots show the correlation coefficient type-average effective connectivity with the cytoarchitectural axis. Asterisks indicate $\mathrm{p}_{\text {spin }}<0.004$, (ie: Bonferroni correction of $\alpha=0.05$ for six two-sided tests).
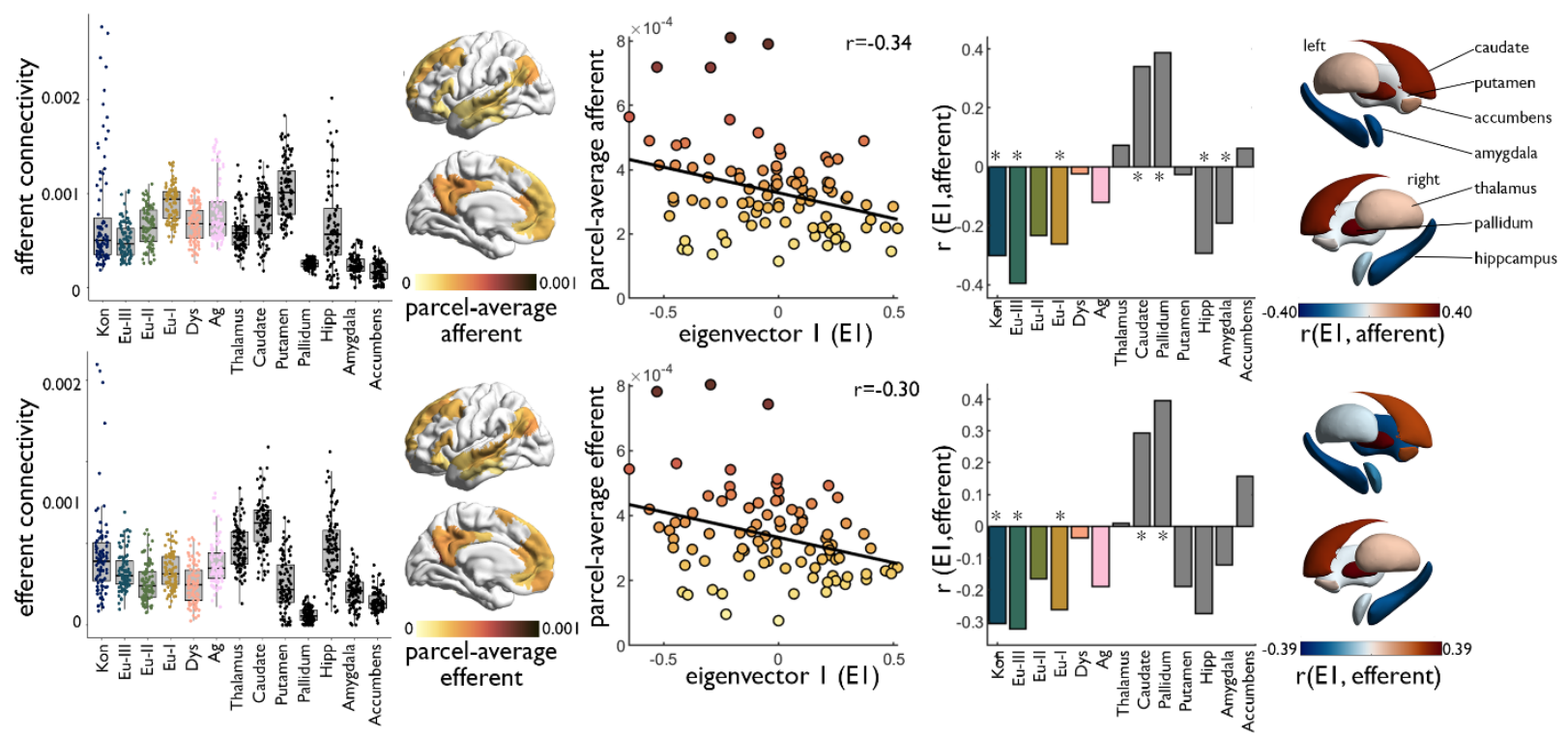

Supplementary Figure 9: Expanded rDCM with non-isocortical regions. Left Boxplots show type-average effective connectivity with the DMN, where each point reflecting a DMN parcel. Centre left Surface plots show the average effective connectivity of each DMN parcel, taken across all non-DMN isocortical parcels. This represents the y-axis in the correlation with parcel-wise values of eigenvector 1 (E1, ie: data-driven cytoarchitectural axis) in the scatterplot. Centre right Bar plots show the correlation coefficient type- or region-average effective connectivity with the cytoarchitectural axis. Asterisks indicate $\mathrm{p}_{\text {spin }}<0.002$ (ie: Bonferroni correction of $\alpha=0.05$ for thirteen two-sided tests). Far right Surface plots show the correlation coefficient of effective connectivity with the cytoarchitectural axis for non-isocortical regions. 
bioRxiv preprint doi: https://doi.org/10.1101/2021.11.22.469533; this version posted November 22, 2021. The copyright holder for this preprint (which was not certified by peer review) is the author/funder, who has granted bioRxiv a license to display the preprint in perpetuity. It is made available under aCC-BY-NC-ND 4.0 International license.

\section{Supplementary Table 1: Roughness of cytoarchitectural axis within DMN subregion}

\begin{tabular}{|c|c|c|c|c|c|c|c|c|c|c|c|c|c|c|}
\hline & & \multicolumn{2}{|c|}{ Parahippocampus } & \multicolumn{2}{|l|}{ IPL } & \multicolumn{2}{|l|}{ MTG } & \multicolumn{2}{|l|}{ IFG } & \multicolumn{2}{|c|}{ Precuneus } & \multicolumn{2}{|l|}{ PFC } & \multirow{2}{*}{$\begin{array}{l}\mathrm{CoV} \\
\%\end{array}$} \\
\hline & & left & right & left & right & left & right & left & right & left & right & left & right & \\
\hline \multicolumn{15}{|c|}{ Amplitude parameters } \\
\hline $\mathrm{R}_{\mathrm{a}}$ & Arithmetic average height & 2.59 & 1.99 & 3.60 & 3.42 & 3.07 & 3.16 & 2.63 & 2.70 & 2.79 & 3.16 & 2.80 & 2.92 & 15 \\
\hline $\mathrm{R}_{\mathrm{q}}$ & Root mean square roughness & 2.90 & 2.36 & 2.78 & 2.62 & 1.99 & 2.57 & 2.64 & 2.15 & 2.95 & 2.98 & 2.55 & 2.62 & 12 \\
\hline $\mathrm{R}_{\mathrm{p}}$ & Maximum height of peaks & 5.43 & 4.32 & 5.45 & 5.45 & 5.43 & 5.45 & 5.16 & 3.67 & 5.48 & 5.44 & 5.37 & 5.35 & 11 \\
\hline $\mathrm{R}_{\mathrm{v}}$ & Maximum depth of valleys & 4.53 & 4.88 & 4.26 & 4.43 & 4.33 & 4.59 & 4.62 & 4.58 & 4.62 & 4.57 & 4.67 & 4.66 & 4 \\
\hline $\mathrm{R}_{\mathrm{pm}}$ & Mean height of peaks & 5.06 & 4.28 & 5.38 & 5.34 & 5.16 & 5.26 & 4.26 & 3.11 & 5.34 & 5.35 & 4.46 & 4.56 & 14 \\
\hline $\mathbf{R}_{\mathrm{vm}}$ & Mean depth of valleys & 4.44 & 4.56 & 3.77 & 3.78 & 2.25 & 3.76 & 4.50 & 4.48 & 4.02 & 4.05 & 4.54 & 4.53 & 16 \\
\hline $\mathrm{k}$ & Solidarity factor & 0.84 & 1.13 & 0.78 & 0.81 & 0.80 & 0.84 & 0.90 & 1.25 & 0.84 & 0.84 & 0.87 & 0.87 & 16 \\
\hline $\mathrm{R}_{\mathrm{sk}}$ & Skewness & -0.27 & 0.40 & -1.09 & -1.11 & -0.99 & -1.00 & 0.65 & 1.00 & -0.28 & -0.60 & 1.01 & 1.11 & 911 \\
\hline $\mathrm{R}_{\mathrm{ku}}$ & Kurtosis & 1.86 & 2.40 & 2.88 & 3.26 & 3.39 & 2.96 & 2.28 & 3.00 & 1.85 & 1.99 & 3.05 & 3.17 & 21 \\
\hline \multicolumn{15}{|c|}{ Spatial parameters } \\
\hline $\mathrm{P}_{\mathrm{c}}$ & Peak count & 4 & 3 & 10 & 11 & 21 & 10 & 7 & 4 & 13 & 7 & 15 & 14 & 54 \\
\hline$S$ & Mean spacing between peaks & 24.10 & 23.25 & 31.68 & 30.59 & 48.25 & 44.62 & 36.38 & 24.16 & 35.91 & 32.91 & 73.60 & 65.36 & 41 \\
\hline $\mathrm{S}_{\mathrm{m}}$ & $\begin{array}{l}\text { Mean spacing between zero } \\
\text { plane }\end{array}$ & 1.32 & 1.34 & 0.92 & 0.90 & 1.02 & 1.06 & 0.86 & 1.02 & 1.13 & 1.08 & 1.04 & 1.12 & 14 \\
\hline n0 & $\begin{array}{l}\text { Number of intersections of } \\
\text { zero plane }\end{array}$ & 180 & 230 & 626 & 421 & 738 & 599 & 1112 & 251 & 898 & 750 & 2110 & 1546 & 73 \\
\hline$r_{p}$ & Mean size of peaks & 21.00 & 19.00 & 22.40 & 15.27 & 15.14 & 19.40 & 36.71 & 18.00 & 19.54 & 32.00 & 46.00 & 38.64 & 41 \\
\hline \multicolumn{15}{|c|}{ Hybrid parameters } \\
\hline$\gamma$ & Mean slope at zero plane & 2.13 & 1.72 & 2.98 & 3.31 & 2.15 & 2.83 & 2.61 & 3.23 & 2.24 & 3.03 & 2.69 & 3.15 & 19 \\
\hline$\Delta_{\mathrm{a}}$ & Mean slope & 0.68 & 0.88 & 0.78 & 0.90 & 0.78 & 0.91 & 0.93 & 0.84 & 0.90 & 0.96 & 0.82 & 0.87 & 9 \\
\hline$\Delta_{\mathrm{q}}$ & Root mean square of slope & 1.27 & 1.48 & 1.47 & 1.69 & 1.39 & 1.61 & 1.70 & 1.52 & 1.64 & 1.75 & 1.49 & 1.67 & 9 \\
\hline$\lambda_{\mathrm{a}}$ & Average wavelength & 11.00 & 10.99 & 17.72 & 19.28 & 15.05 & 17.99 & 15.35 & 14.24 & 15.84 & 19.09 & 14.38 & 16.00 & 18 \\
\hline$\lambda_{\mathrm{q}}$ & $\begin{array}{l}\text { Root mean square of } \\
\text { wavelength }\end{array}$ & 23.14 & 21.87 & 25.77 & 27.81 & 17.38 & 26.02 & 28.17 & 20.48 & 30.31 & 32.73 & 23.92 & 27.47 & 17 \\
\hline$S_{\mathrm{f}}$ & Stepness factor & 3.41 & 2.67 & 3.32 & 3.08 & 3.14 & 3.35 & 2.26 & 2.77 & 3.17 & 3.41 & 2.90 & 3.25 & 11 \\
\hline $\mathrm{W}_{\mathrm{f}}$ & Waviness factor & 652 & 589 & 1247 & 979 & 2120 & 1269 & 2042 & 587 & 1830 & 1415 & 4941 & 3736 & 74 \\
\hline
\end{tabular}

IPL: inferior parietal lobule. MTG: middle temporal gyrus. IFG: inferior frontal gyrus. PFC: prefrontal cortex. CoV: coefficient of variation.

Supplementary Table 2: Cortical types by functional network.

\begin{tabular}{l|cccccc|c} 
& Kon & Eu-III & Eu-II & Eu-I & Dys & Ag & $\begin{array}{c}\text { Total } \\
\text { vertices }\end{array}$ \\
\hline Visual & 0.29 & 0.41 & 0.17 & 0.10 & 0.00 & 0.03 & 2750 \\
Somatomotor & 0.10 & 0.54 & 0.31 & 0.04 & 0.00 & 0.01 & 3751 \\
DAN & 0.00 & 0.40 & 0.53 & 0.06 & 0.00 & 0.00 & 2188 \\
VAN & 0.02 & 0.18 & 0.50 & 0.13 & 0.08 & 0.09 & 2285 \\
Limbic & 0.00 & 0.24 & 0.28 & 0.11 & 0.26 & 0.10 & 1426 \\
Frontoparietal & 0.00 & 0.18 & 0.56 & 0.23 & 0.01 & 0.04 & 2314 \\
Default mode & 0.00 & 0.32 & 0.31 & 0.28 & 0.02 & 0.07 & 3765 \\
\hline Total vertices & 1218 & 6400 & 6805 & 2572 & 648 & 836 &
\end{tabular}

Note: proportions are provided relative to the functional network (ie: $29 \%$ of the visual network is Kon).

Kon=koniocortical. Eu=eulaminate. Dys=dysgranular. Ag=agranular. DAN=dorsal attention network. VAN=ventral attention network. 
bioRxiv preprint doi: https://doi.org/10.1101/2021.1122.469533; this version posted November 22, 2021. The copyright holder for this preprint (which was not certified by peer review) is the author/funder, who has granted bioRxiv a license to display the preprint in perpetuity. It is made available under aCC-BY-NC-ND 4.0 International license.

Supplementary Table 3: Key statistics in primary and replication analyses for navigation efficiency

\begin{tabular}{|c|c|c|c|}
\hline Statistical test & Type/network & MICs & HCP \\
\hline \multirow{6}{*}{$\begin{array}{l}\text { Correlation with data- } \\
\text { driven cytoarchitectural } \\
\text { axis (averaged by type, } \\
\text { calculated for non-DMN } \\
\text { targets) }\end{array}$} & Kon & $\mathrm{r}=-0.63, \mathrm{p}<0.001$ & $\mathrm{r}=-0.51, \mathrm{p}<0.001$ \\
\hline & Eu-III & $\mathrm{r}=-0.55, \mathrm{p}<0.001$ & $\mathrm{r}=-0.55, \mathrm{p}<0.001$ \\
\hline & Eu-III & $\mathrm{r}=-0.33, \mathrm{p}=0.015$ & $\mathrm{r}=-0.06, \mathrm{p}=0.615$ \\
\hline & Eu-I & $r=-0.29, p=0.062$ & $\mathrm{r}=0.11, \mathrm{p}=0.707$ \\
\hline & Dys & $\mathrm{r}=0.08, \mathrm{p}=0.325$ & $r=0.06, p=0.369$ \\
\hline & $\mathrm{Ag}$ & $\mathrm{r}=0.12, \mathrm{p}=0.185$ & $\mathrm{r}=0.22, \mathrm{p}=0.072$ \\
\hline \multirow{7}{*}{$\begin{array}{l}\text { Divergence from } \\
\text { balanced model }\end{array}$} & Visual & $\mathrm{KL}=0.015, \mathrm{p}=0.364$ & $\mathrm{KL}=0.017, \mathrm{p}=0.077$ \\
\hline & Somatomotor & $\mathrm{KL}=0.006, \mathrm{p}=0.288$ & $\mathrm{KL}=0.044, \mathrm{p}=0.705$ \\
\hline & DAN & $\mathrm{KL}=0.023, \mathrm{p}=0.957$ & $\mathrm{KL}=0.056, \mathrm{p}=0.819$ \\
\hline & VAN & $\mathrm{KL}=0.005, \mathrm{p}=0.472$ & $\mathrm{KL}=0.019, \mathrm{p}=0.541$ \\
\hline & Limbic & $\mathrm{KL}=0.034, \mathrm{p}=0.761$ & $\mathrm{KL}=0.043, \mathrm{p}=0.375$ \\
\hline & Frontoparietal & $\mathrm{KL}=0.005, \mathrm{p}=0.683$ & $\mathrm{KL}=0.017, \mathrm{p}=0.491$ \\
\hline & DMN & $\mathrm{KL}=0.002, \mathrm{p}=0.107$ & $\mathrm{KL}=0.010, \mathrm{p}=0.281$ \\
\hline
\end{tabular}


Supplementary Table 4: Key statistics in primary and replication analyses for cortical only rDCM

\begin{tabular}{|c|c|c|c|c|c|}
\hline \multirow{2}{*}{ Statistical test } & \multirow{2}{*}{ Type/network } & \multicolumn{2}{|c|}{ MICs } & \multicolumn{2}{|c|}{ HCP } \\
\hline & & Afferent & Efferent & Afferent & Efferent \\
\hline \multicolumn{2}{|c|}{$\begin{array}{l}\text { Multiple linear regression model of effective } \\
\text { connectivity by cytoarchitecture }\end{array}$} & $\begin{array}{l}\mathrm{R}^{2}=0.24 \\
\mathrm{p}<0.001\end{array}$ & $\begin{array}{l}\mathrm{R}^{2}=0.06 \\
\mathrm{p}<0.001\end{array}$ & $\begin{array}{l}\mathrm{R}^{2}=0.22 \\
\mathrm{p}<0.001\end{array}$ & $\begin{array}{l}\mathrm{R}^{2}=0.013 \\
\mathrm{p}<0.001\end{array}$ \\
\hline \multirow[t]{7}{*}{$\begin{array}{l}\text { Correlation with data-driven } \\
\text { cytoarchitectural axis }\end{array}$} & All non-DMN & $\begin{array}{l}r=-0.41 \\
p<0.001\end{array}$ & $\begin{array}{l}r=-0.05 \\
p=0.395\end{array}$ & $\begin{array}{l}\mathrm{r}=-0.31 \\
\mathrm{p}<0.001\end{array}$ & $\begin{array}{l}r=-0.24 \\
p<0.001\end{array}$ \\
\hline & Kon & $\begin{array}{l}\mathrm{r}=-0.36 \\
\mathrm{p}=0.001\end{array}$ & $\begin{array}{l}\mathrm{r}=-0.06 \\
\mathrm{p}=0.129\end{array}$ & $\begin{array}{l}\mathrm{r}=-0.23 \\
\mathrm{p}=0.001\end{array}$ & $\begin{array}{l}\mathrm{r}=-0.25 \\
\mathrm{p}=0.001\end{array}$ \\
\hline & Eu-III & $\begin{array}{l}\mathrm{r}=-0.45 \\
\mathrm{p}<0.001\end{array}$ & $\begin{array}{l}r=0.00 \\
p=0.185\end{array}$ & $\begin{array}{l}\mathrm{r}=-0.35 \\
\mathrm{p}<0.001\end{array}$ & $\begin{array}{l}r=-0.23, \\
p<0.001\end{array}$ \\
\hline & Eu-III & $\begin{array}{l}\mathrm{r}=-0.21 \\
\mathrm{p}=0.001\end{array}$ & $\begin{array}{l}\mathrm{r}=0.16 \\
\mathrm{p}=0.817\end{array}$ & $\begin{array}{l}\mathrm{r}=-0.22 \\
\mathrm{p}=0.001\end{array}$ & $\begin{array}{l}\mathrm{r}=-0.11 \\
\mathrm{p}=0.014\end{array}$ \\
\hline & Eu-I & $\begin{array}{l}\mathrm{r}=-0.44 \\
\mathrm{p}<0.001\end{array}$ & $\begin{array}{l}\mathrm{r}=0.01 \\
\mathrm{p}=0.613\end{array}$ & $\begin{array}{l}\mathrm{r}=-0.22 \\
\mathrm{p}=0.028\end{array}$ & $\begin{array}{l}r=-0.26 \\
p=0.001\end{array}$ \\
\hline & Dys & $\begin{array}{l}r=-0.23 \\
p=0.004\end{array}$ & $\begin{array}{l}r=0.04 \\
p=0.410\end{array}$ & $\begin{array}{l}r=-0.20 \\
p=0.029\end{array}$ & $\begin{array}{l}r=-0.07 \\
p=0.129\end{array}$ \\
\hline & $\mathrm{Ag}$ & $\begin{array}{l}r=-0.37 \\
p=0.004\end{array}$ & $\begin{array}{l}\mathrm{r}=0.05 \\
\mathrm{p}=0.225\end{array}$ & $\begin{array}{l}r=-0.20 \\
p=0.059\end{array}$ & $\begin{array}{l}r=-0.29 \\
p<0.001\end{array}$ \\
\hline \multirow{6}{*}{$\begin{array}{l}\text { Average type-specific } \\
\text { connectivity (effective } \\
\text { connectivity } \times 10^{-3} \text { ) }\end{array}$} & Kon & $\begin{array}{l}0.53 \pm 0.47 \\
p=0.311\end{array}$ & $\begin{array}{l}0.62 \pm 0.49 \\
p=0.126\end{array}$ & $\begin{array}{l}0.53 \pm 0.35 \\
p=0.012\end{array}$ & $\begin{array}{l}0.42 \pm 0.28 \\
\mathrm{p}=0.027\end{array}$ \\
\hline & Eu-III & $\begin{array}{l}0.35 \pm 0.13 \\
p=0.986\end{array}$ & $\begin{array}{l}0.50 \pm 0.22 \\
p=0.164\end{array}$ & $\begin{array}{l}0.27 \pm 0.12 \\
p=0.864\end{array}$ & $\begin{array}{l}0.26 \pm 0.15 \\
p=0.609\end{array}$ \\
\hline & Eu-III & $\begin{array}{l}0.47 \pm 0.17 \\
p=0.364\end{array}$ & $\begin{array}{l}0.38 \pm 0.19 \\
p=0.954\end{array}$ & $\begin{array}{l}0.25 \pm 0.10 \\
p=0.984\end{array}$ & $\begin{array}{l}0.24 \pm 0.10 \\
p=0.942\end{array}$ \\
\hline & Eu-I & $\begin{array}{l}0.74 \pm 0.19 \\
p<0.001\end{array}$ & $\begin{array}{l}0.50 \pm 0.24 \\
p=0.258\end{array}$ & $\begin{array}{l}0.37 \pm 0.16 \\
p=0.030\end{array}$ & $\begin{array}{l}0.32 \pm 0.15 \\
p=0.078\end{array}$ \\
\hline & Dys & $\begin{array}{l}0.47 \pm 0.19 \\
\mathrm{p}=0.522\end{array}$ & $\begin{array}{l}0.48 \pm 0.25 \\
\mathrm{p}=0.508\end{array}$ & $\begin{array}{l}0.27 \pm 0.10 \\
p=0.613\end{array}$ & $\begin{array}{l}0.26 \pm 0.15 \\
p=0.522\end{array}$ \\
\hline & $\mathrm{Ag}$ & $\begin{array}{l}0.54 \pm 0.33 \\
p=0.337\end{array}$ & $\begin{array}{l}0.63 \pm 0.33 \\
\mathrm{p}=0.131\end{array}$ & $\begin{array}{l}0.43 \pm 0.29 \\
\mathrm{p}=0.077\end{array}$ & $\begin{array}{l}0.32 \pm 0.20 \\
p=0.267\end{array}$ \\
\hline \multirow[t]{7}{*}{$\begin{array}{l}\text { Divergence from balanced } \\
\text { model }\end{array}$} & Visual & $\begin{array}{l}\mathrm{KL}=0.01 \\
\mathrm{p}=0.038\end{array}$ & $\begin{array}{l}\mathrm{KL}=0.04 \\
\mathrm{p}=0.197\end{array}$ & $\begin{array}{l}\mathrm{KL}=0.06 \\
\mathrm{p}=0.445\end{array}$ & $\begin{array}{l}\mathrm{KL}=0.05 \\
\mathrm{p}=0.196\end{array}$ \\
\hline & Somatomotor & $\begin{array}{l}\mathrm{KL}=0.07 \\
\mathrm{p}=0.956\end{array}$ & $\begin{array}{l}\mathrm{KL}=0.07 \\
\mathrm{p}=0.896\end{array}$ & $\begin{array}{l}\mathrm{KL}=0.05, \\
\mathrm{p}=0.783\end{array}$ & $\begin{array}{l}\mathrm{KL}=0.13, \\
\mathrm{p}=0.996\end{array}$ \\
\hline & DAN & $\begin{array}{l}\mathrm{KL}=0.03 \\
\mathrm{p}=0.657\end{array}$ & $\begin{array}{l}\mathrm{KL}=0.08 \\
\mathrm{p}=0.999\end{array}$ & $\begin{array}{l}\mathrm{KL}=0.02 \\
\mathrm{p}=0.388\end{array}$ & $\begin{array}{l}\mathrm{KL}=0.07 \\
\mathrm{p}=0.724\end{array}$ \\
\hline & VAN & $\begin{array}{l}\mathrm{KL}=0.20 \\
\mathrm{p}=1\end{array}$ & $\begin{array}{l}\mathrm{KL}=0.17, \\
\mathrm{p}=1\end{array}$ & $\begin{array}{l}\mathrm{KL}=0.15, \\
\mathrm{p}=1\end{array}$ & $\begin{array}{l}\mathrm{KL}=0.24, \\
\mathrm{p}=1\end{array}$ \\
\hline & Limbic & $\begin{array}{l}\mathrm{KL}=0.06 \\
\mathrm{p}=1\end{array}$ & $\begin{array}{l}\mathrm{KL}=0.14, \\
\mathrm{p}=1\end{array}$ & $\begin{array}{l}\mathrm{KL}=0.03, \\
\mathrm{p}=0.517\end{array}$ & $\begin{array}{l}\mathrm{KL}=0.03 \\
\mathrm{p}=0.054\end{array}$ \\
\hline & Frontoparietal & $\begin{array}{l}\mathrm{KL}=0.04 \\
\mathrm{p}=0.479\end{array}$ & $\begin{array}{l}\mathrm{KL}=0.07 \\
\mathrm{p}=0.718\end{array}$ & $\begin{array}{l}\mathrm{KL}=0.06 \\
\mathrm{p}=0.804\end{array}$ & $\begin{array}{l}\mathrm{KL}=0.08 \\
\mathrm{p}=0.597\end{array}$ \\
\hline & $\mathrm{DMN}$ & $\begin{array}{l}\mathrm{KL}=0.04 \\
\mathrm{p}=0.938\end{array}$ & $\begin{array}{l}\mathrm{KL}=0.02 \\
\mathrm{p}=0.043\end{array}$ & $\begin{array}{l}\mathrm{KL}=0.06 \\
\mathrm{p}=0.995\end{array}$ & $\begin{array}{l}\mathrm{KL}=0.03, \\
\mathrm{p}=0.013\end{array}$ \\
\hline
\end{tabular}


Supplementary Table 5: Key statistics in primary and replication analyses for extended rDCM

\begin{tabular}{|c|c|c|c|c|c|}
\hline \multirow{2}{*}{ Statistical test } & \multirow{2}{*}{ Type/network } & \multicolumn{2}{|c|}{ MICs } & \multicolumn{2}{|c|}{ HCP } \\
\hline & & Afferent & Efferent & Afferent & Efferent \\
\hline \multicolumn{2}{|c|}{$\begin{array}{l}\text { Multiple linear regression model of effective } \\
\text { connectivity by cytoarchitecture }\end{array}$} & $\begin{array}{l}\mathrm{R}^{2}=0.46 \\
\mathrm{p}<0.001\end{array}$ & $\begin{array}{l}\mathrm{R}^{2}=0.50 \\
\mathrm{p}<0.001\end{array}$ & $\begin{array}{l}\mathrm{R}^{2}=0.46 \\
\mathrm{p}<0.001\end{array}$ & $\begin{array}{l}\mathrm{R}^{2}=0.48 \\
\mathrm{p}<0.001\end{array}$ \\
\hline \multirow[t]{27}{*}{$\begin{array}{l}\text { Correlation with data-driven } \\
\text { cytoarchitectural axis }\end{array}$} & $\begin{array}{l}\text { All non-DMN } \\
\text { isocortex }\end{array}$ & $\begin{array}{l}\mathrm{r}=-0.40 \\
\mathrm{p}<0.001\end{array}$ & $\begin{array}{l}\mathrm{r}=0.00 \\
\mathrm{p}=0.325\end{array}$ & $\begin{array}{l}\mathrm{r}=-0.34 \\
\mathrm{p}<0.001\end{array}$ & $\begin{array}{l}\mathrm{r}=-0.30 \\
\mathrm{p}<0.001\end{array}$ \\
\hline & Kon & $\mathrm{r}=-0.41$, & $\mathrm{r}=-0.15$, & $\mathrm{r}=-0.30$, & $\mathrm{r}=-0.31$, \\
\hline & & $\mathrm{p}<0.001$ & $p=0.026$ & $\mathrm{p}=0.004$ & $\mathrm{p}=0.001$ \\
\hline & Eu-III & $\mathrm{r}=-0.43$ & $\mathrm{r}=0.03$ & $\mathrm{r}=-0.40$ & $\mathrm{r}=-0.32$ \\
\hline & & $\mathrm{p}<0.001$ & $\mathrm{p}=0.280$ & $\mathrm{p}<0.001$ & $\mathrm{p}<0.001$ \\
\hline & Eu-III & $\mathrm{r}=-0.14$ & $\mathrm{r}=0.22$ & $\mathrm{r}=-0.23$ & $\mathrm{r}=-0.17$ \\
\hline & & $\mathrm{p}=0.025$ & $\mathrm{p}=0.975$ & $\mathrm{p}=0.011$ & $\mathrm{p}=0.033$ \\
\hline & Eu-I & $\mathrm{r}=-0.41$ & $\mathrm{r}=0.09$ & $\mathrm{r}=-0.26$ & $\mathrm{r}=-0.26$ \\
\hline & & $\mathrm{p}<0.001$ & $\mathrm{p}=0.673$ & $\mathrm{p}=0.007$ & $\mathrm{p}=0.004$ \\
\hline & Dys & $\mathrm{r}=-0.11$, & $\mathrm{r}=0.02$ & $r=-0.03$ & $\mathrm{r}=-0.04$ \\
\hline & & $\mathrm{p}=0.026$ & $\mathrm{p}=0.293$ & $\mathrm{p}=0.310$ & $\mathrm{p}=0.240$ \\
\hline & $\mathrm{Ag}$ & $\mathrm{r}=-0.21$ & $\mathrm{r}=0.05$ & $\mathrm{r}=-0.12$ & $\mathrm{r}=-0.10$ \\
\hline & & $\mathrm{p}=0.019$ & $p=0.656$ & $\mathrm{p}=0.180$ & $\mathrm{p}=0.061$ \\
\hline & Thalamus & $\mathrm{r}=-0.02$ & $\mathrm{r}=-0.15$ & $\mathrm{r}=0.072$ & $\mathrm{r}=0.01$ \\
\hline & & $\mathrm{p}=0.383$ & $\mathrm{p}=0.099$ & $\mathrm{p}=0.100$ & $\mathrm{p}=0.250$ \\
\hline & Caudate & $\mathrm{r}=0.50$ & $\mathrm{r}=0.43$ & $\mathrm{r}=0.34$ & $\mathrm{r}=0.29$ \\
\hline & & $\mathrm{p}<0.001$ & $\mathrm{p}<0.001$ & $\mathrm{p}<0.001$ & $\mathrm{p}<0.001$ \\
\hline & Pallidum & $\mathrm{r}=0.05$ & $\mathrm{r}=0.45$ & $\mathrm{r}=0.39$ & $\mathrm{r}=0.40$ \\
\hline & & $\mathrm{p}=0.393$ & $\mathrm{p}<0.001$ & $\mathrm{p}<0.001$ & $\mathrm{p}<0.001$ \\
\hline & Putamen & $\mathrm{r}=-0.26$ & $\mathrm{r}=-0.33$ & $\mathrm{r}=-0.03$ & $\mathrm{r}=-0.19$ \\
\hline & & $\mathrm{p}=0.002$ & $\mathrm{p}=0.002$ & $\mathrm{p}=0.521$ & $\mathrm{p}=0.056$ \\
\hline & Hippocampus & $\mathrm{r}=-0.19$ & $\mathrm{r}=-0.15$ & $\mathrm{r}=-0.29$ & $\mathrm{r}=-0.27$ \\
\hline & & $\mathrm{p}=0.038$ & $\mathrm{p}=0.041$ & $\mathrm{p}=0.003$ & $\mathrm{p}=0.047$ \\
\hline & Amygdala & $\mathrm{r}=-0.07$ & $\mathrm{r}=0.16$ & $\mathrm{r}=-0.19$ & $\mathrm{r}=-0.12$ \\
\hline & & $\mathrm{p}=0.153$ & $\mathrm{p}=0.131$ & $\mathrm{p}=0.007$ & $\mathrm{p}=0.033$ \\
\hline & Accumbens & $\mathrm{r}=0.14$ & $\mathrm{r}=0.32$ & $\mathrm{r}=0.06$ & $\mathrm{r}=0.16$ \\
\hline & & $\mathrm{p}=0.050$ & $\mathrm{p}=0.001$ & $\mathrm{p}=0.187$ & $\mathrm{p}=0.018$ \\
\hline \multirow{10}{*}{$\begin{array}{l}\text { Average type-specific } \\
\text { connectivity (effective } \\
\text { connectivity } \times 10^{-3} \text { ) }\end{array}$} & Kon & $0.73 \pm 0.59$ & $0.85 \pm 0.55$ & $0.51 \pm 0.33$ & $0.52 \pm 0.32$ \\
\hline & & $\mathrm{p}=0.305$ & $\mathrm{p}=0.005$ & $\mathrm{p}=0.028$ & $\mathrm{p}=0.028$ \\
\hline & Eu-III & $0.50 \pm 0.19$ & $0.66 \pm 0.24$ & $0.24 \pm 0.16$ & $0.33 \pm 0.17$ \\
\hline & & $\mathrm{p}=0.980$ & $\mathrm{p}=0.147$ & $\mathrm{p}=0.388$ & $\mathrm{p}=0.482$ \\
\hline & Eu-III & $\begin{array}{l}0.64 \pm 0.21 \\
p=0.380\end{array}$ & $\begin{array}{l}0.52 \pm 0.23 \\
p=0.997\end{array}$ & $\begin{array}{l}0.31 \pm 0.13 \\
\mathrm{p}=0.838\end{array}$ & $\begin{array}{l}0.30 \pm 0.13 \\
p=0.839\end{array}$ \\
\hline & Eu-I & $0.90 \pm 0.19$ & $0.66 \pm 0.22$ & $0.39 \pm 0.20$ & $0.38 \pm 0.20$ \\
\hline & & $\mathrm{p}<0.001$ & $\mathrm{p}=0.231$ & $\mathrm{p}=0.102$ & $\mathrm{p}=0.109$ \\
\hline & Dys & $\begin{array}{l}0.67 \pm 0.19 \\
p=0.479\end{array}$ & $\begin{array}{l}0.51 \pm 0.25 \\
p=0.760\end{array}$ & $\begin{array}{l}0.19 \pm 0.07 \\
p=0.914\end{array}$ & $\begin{array}{l}0.21 \pm 0.10 \\
p=0.821\end{array}$ \\
\hline & $\mathrm{Ag}$ & $0.80 \pm 0.33$ & $0.76 \pm 0.27$ & $0.24 \pm 0.16$ & $0.27 \pm 0.17$ \\
\hline & & $\mathrm{p}=0.213$ & $\mathrm{p}=0.189$ & $\mathrm{p}=0.865$ & $\mathrm{p}=0.712$ \\
\hline
\end{tabular}

